\title{
PERTURBATIONS OF DISCONTINUOUS SOLUTIONS OF NON-LINEAR SYSTEMS OF DIFFERENTIAL EQUATIONS.
}

By

NORMAN LEVINSON')

of CAmbridge, U. S. A.

1. The study of the solutions of the system

$$
\frac{d x_{i}}{d t}=X_{i}\left(x_{1}, \ldots, x_{n}, t, \varepsilon\right), \quad i=1,2, \ldots n,
$$

where the $X_{i}$ are regular functions of $\varepsilon$ for small $\varepsilon$ is classical. More recently nonlinear systems like (1.0) have been studied when one or more of the $X_{i}$ has a pole at $\varepsilon=0$, or what is equivalent, where $\varepsilon$ or some power of $\varepsilon$ occurs as the coefficient of the left member of one or more of the equations $(1.0),[1,2,3,4,5,6]$. In this case the system when $\varepsilon=0$ is of lower order than when $\varepsilon \neq 0$. In the studies $[2,4,6]$ it is assumed that the system has a solution with a continuous derivative in case $\varepsilon=0$ and conditions are given for this to be the case when $\varepsilon \neq 0$.

In applied mathematics there are cases where the system has only discontinuous "solutions" when $\varepsilon=0$ and yet is known empirically that when $\varepsilon>0$ the system has a continuous solution which approaches the discontinuous one as $\varepsilon \rightarrow 0$. This fact has been exploited by the Russian school of non-linear mechanics. Here a rigorous treatment will be given for a case where the system has a discontinuous "solution" when $\varepsilon=0$. The main result has already been announced, without proof, [3]. Since [3] has appeared, a system with a discontinuous solution has been treated [5] by Tihonov. In Tihonov's treatment the "jump ares" instead of being solutions of (2.2) must be straight lines. Also the existence of derivatives with respect to initial values is not considered.

The specific system we shall consider here is

\footnotetext{
1 John Simon Guggenheim Memorial Fellow on leave from the Massachusetts Institute of Technology.
} 


$$
\frac{d x_{i}}{d t}=f_{i} \frac{d u}{d t}+\varphi_{i}, \quad i=1,2, \ldots, n, \quad \varepsilon \frac{d^{2} \dot{u}}{d t^{2}}+g \frac{d u}{d t}+h=0 .
$$

Here $f_{i}, \varphi_{i}, g$ and $h$ are functions of $x_{1}, x_{2}, \ldots, x_{n}, u, t$ and $\varepsilon$. They are continuous in $\varepsilon$ for small $\varepsilon \geqq 0$. It will be convenient to use a vector notation and to denote the vector with components $x_{i}$ by $x$, the vector with components $f_{i}$ by $f$, and $\varphi_{i}$ by $\varphi$. Thus we can write (1.1) as

$$
\frac{d x}{d t}=f \frac{d u}{d t}+\varphi, \quad \varepsilon \frac{d^{2} u}{d t^{2}}+g \frac{d u}{d t}+h=0
$$

where the first equation of (1.2) is a vector equation. The vector $f$ is $f(x, u, t, \varepsilon)$ and similarly for the vector $\varphi$ and the scalars $g$ and $h$.

By the degenerate system we shall mean (1.2) with $\varepsilon=0$. We observe that the degenerate system is of lower order than (1.2). We shall write the degenerate system as

$$
\frac{d y}{d t}=f \frac{d v}{d t}+\varphi, \quad g \frac{d v}{d t}+h=0
$$

where $y$ is a vector with components $y_{1}, \ldots, y_{n}$ and $v$ is a scalar. In (1.3) $f$ is $f(y, v, t, 0)$ and similarly for $\varphi, g$ and $h$. Let us now consider a solution of (1.3) as a curve in the $n+2$ dimensional space $(y, v, t)$. We assume that such a solution starts at a point, $A$. We observe that when the solution reaches a point on the hypersurface $g(y, v, t, 0)$ $=0$, a singular situation may prevail with regard to $d v / d t$.

This situation can be seen clearly by taking a very simple special case. We take the van der Pol equation with a change of time scale which may be written as

$$
\varepsilon \frac{d^{2} u}{d t^{2}}+\left(u^{2}-1\right) \frac{d u}{d t}+u=0
$$

and we consider the related degenerate equation

$$
\left(v^{2}-1\right) \frac{d v}{d t}+v=0 \text {. }
$$

The solution $v(t)$ of (1.5) which at $t=0$ satisfies $v(0)>1$ is readily obtainable. However all we need observe is that since

$$
\frac{d v}{d t}=-\frac{v}{v^{2}-1}<0 .
$$

$v$ is decreasing. Thus for $v \geqq 1$ 


$$
\frac{d v}{d t}<-\frac{1}{v^{2}(0)-1}
$$

and after a finite elapse of $t$ we have for some $t=t_{1}, v\left(t_{1}\right)=1$. As $t \rightarrow t_{1}-0, \frac{d v}{d t} \rightarrow-\infty$. That is for $v=1+0, d v / d t=-\infty$. Any attempt to continue the solution, as a continuous function of $t$, beyond $t=t_{1}$, fails since for $v=1-0, d v / d t=+\infty$. Thus the solution cannot pass continuously from above $v=1$ to below $v=1$. Moreover since $v=1$ is obviously not a solution of (1.5) the solution cannot be continued as $v=1$. We note that $v=1$ here corresponds to $g=0$ in (1.3).

If we turn to (1.4) with $\varepsilon>0$ we see that the line $u=1$ offers no special difficulty. Thus a solution $u(t)$ of (1.4) obviously can be continued beyond a point where $u=1+0$. Let $u=1+0$ when $t=t_{1}-0$ and let us integrate (1.4) from $t_{1}-\delta$ to $t_{1}+\delta$ where $\delta>0$ is small. We find

$$
\left.\left.\varepsilon \frac{d u}{d t}\right]_{t_{1}-\delta}^{t_{1}+\delta}+\left(\frac{u^{3}}{3}-u\right)\right]_{t_{1}-\delta}^{t_{1}+\delta}+\int_{t_{1}-\delta}^{t_{1}+\delta} u d t=0 .
$$

Now let us proceed heuristically. If as $\varepsilon \rightarrow 0$ we assume $\frac{d u\left(t_{1} \pm \delta\right)}{d t}$ approach finite limiting values and if we assume that $|u|$ remains uniformly bounded in the range $\left(t_{1}-\delta, t_{1}+\delta\right)$ then we get

$$
\left.\left(\frac{u^{3}}{3}-u\right)\right]_{t_{1}-\delta}^{t_{1}+\delta}=O(\delta) .
$$

Now letting $\delta \rightarrow 0$ and recalling that $u\left(t_{1}-0\right)=1$, we get

$$
\frac{1}{3} u^{3}\left(t_{1}+0\right)-u\left(t_{1}+0\right)+\frac{2}{3}=0 \text {. }
$$

Solving this last equation we get either $u\left(t_{1}+0\right)=1$ or $u\left(t_{1}+\theta\right)=-2$. The value 1 we discard on the basis of our experience with (1.5) and we are led to investigate further the possibility $u\left(t_{1}+0\right)=-2$. Actually (1.4) can be investigated directly [1] and it is indeed found that as $\varepsilon \rightarrow 0$, solutions of (1.4) on reaching $u=1+0$ tend to jump to $u=-2$. We shall not pursue this intuitive discussion further but rather proceed in $\S 2$ to give a definition of a solution of (1.3) which may be discontinuous. The definition will be justified because we shall show that as $\varepsilon \rightarrow 0$ solutions of (1.2) tend to solutions, as we define them here, of (1.3).

As was observed in [3] the system (1.2) includes as a special case the system

$$
\frac{d x}{d t}=H(x, w, t, \varepsilon), \quad \varepsilon \frac{d w}{d t}=G(x, w, t, \varepsilon)
$$


where $x$ and $H$ are vectors and $H$ and $G$ are continuous in $\varepsilon$ for small $\varepsilon \geqq 0$. $G$ and $w$ are scalars. With $\varepsilon$ not appearing on the right this is the system treated by Tihonov [5]. In case the right members of (1.6) are not linear in $w$ the system can be brought to the form (1.2) (with $f \equiv 0$ ) simply by differentiating the last equation with respect to $t$. On the other hand if the right members of (1.6) are linear in $w$ then, if we introduce the variable $u$ given by $\frac{d u}{d t}=w$, the system (1.6) assumes the form

$$
\frac{d x}{d t}=f(x, t, \varepsilon) \frac{d u}{d t}+\varphi(x, t, \varepsilon) \quad \varepsilon \frac{d^{2} u}{d t^{2}}+g(x, t, \varepsilon) \frac{d u}{d t}+h(x, t, \varepsilon)=0
$$

which is a special case of (1.2).

2. We shall now give the definition of a solution of (1.3). We consider the solution as a curve, $S_{0}$, in the $n+2$ dimensional space $(y, v, t)$. At the point $A$ let $t=\alpha$ and $g>0$. For $\alpha \leqq t<\tau_{1}$, let $(y(t), v(t))$ be a solution of (1.3) and let

$$
g(y(t), v(t), t, 0)>0, \quad \alpha \leqq t<\tau_{1} .
$$

As $t \rightarrow \tau_{1}-0$ let $g \rightarrow 0$. The point $\left(y\left(\tau_{1}-0\right), v\left(\tau_{1}-0\right), \tau_{1}\right)$ we denote by $B_{1}$. We shall denote $v\left(\tau_{1}-0\right)$ by $v_{B}$ and $y\left(\tau_{1}-0\right)$ by $y_{B} . A B_{1}$ is an arc of $S_{0}$. We assume that at $B_{1}$

$$
I=\sum_{i=1}^{n} \frac{\partial g}{\partial y_{i}} f_{i}+\frac{\partial g}{\partial v} \neq 0
$$

Here $I=I_{B}$. The next arc of $S_{0}$ is $B_{1} C_{1}$ where $B_{1} C_{1}$ is a curve $y(v)$ in the hyperplane $t=\tau_{1}$ which satisfies the vector equations

$$
\frac{d y}{d v}=f\left(y, v, \tau_{1}, 0\right) .
$$

(The system (2.2) is (1.3) with $t$ held constant and with the last equation of (1.3) omitted). The solution of (2.2) starts at $B_{1}$. We then consider (2.2) for increasing or decreasing $v$ according as $h$ at $B$ is respectively negative or positive. We assume that the solution of (2.2) can be continued for increasing or decreasing $v$, depending as we have seen on the sign of $h$ at $B$, until we reach the first value of $v \neq v_{B}$ for which

$$
\int_{v_{B}}^{v} g\left(y(v), v, \tau_{1}, 0\right) d v=0 .
$$

This value of $v$ we call $v_{C}$ and the point $C_{1}$ is given by $\left(y\left(v_{C}\right), v_{C}, \tau_{1}\right)$. At $C_{1}$ we assume

$$
g\left(y\left(v_{C}\right), v_{C}, \tau_{1}, 0\right)=g_{C}>0 .
$$

On the basis of remarks already made we have 


$$
\left(v_{C}-v_{B}\right) h_{B}<0
$$

where $h_{B}=h\left(y_{B}, v_{B}, \tau_{1}, 0\right)$. (The integral corresponding to (2.3) in the case of the van der Pol equation we considered above is simply

$$
\int_{1}^{v}\left(v^{2}-1\right) d v=0
$$

and since $h$ at $B_{1}$ (where $v=1$ ) is positive we must take $v$ decreasing. Thus we see that here $v_{C}=-2$.)

At $C_{1}$ we return to the system (1.3) and consider the solution with initial values at $C_{1}$ and with $t$ increasing. We assume that this solution can be continued with $g>0$ until $t \rightarrow \tau_{2}-0$ where $g \rightarrow 0$. From this point which we denote by $B_{2}$ we assume we can proceed in the manner already indicated at $B_{1}$ with $v$ increasing or decreasing depending on the sign of $h$ at $B_{2}$. Proceeding in this way the solution $S_{0}$ is defined geometrically as $A B_{1} C_{1} B_{2} C_{2} \ldots B_{N} C_{N} A^{\prime}$ where $A^{\prime}$ is an ordinary point (i. e. one where $g>0$ ). We assume that (1.3) has a solution $S_{0}$ as just defined for $\alpha \leqq t \leqq \beta$. We assume further that there exists an open set $R$ in the $n+2$ dimensional space $(y, v, t)$ containing the curve $S_{0}$ such that $f(y, v, t, \varepsilon), \varphi, g$ and $h$ and their first order partial derivatives with respect to $y_{i}, v$ and $t$ are uniformly continuous and bounded as functions of $y, v, t$ and $\varepsilon$ when $(y, v, t)$ is in $R$ and $\varepsilon \geqq 0$ is small. We shall also assume that $g$ and $h$ have second order partial derivatives continuous in $R$ and for small $\varepsilon$ although this assumption can be avoided.

We see that $S_{0}$ considered as a curve (but not as a function of $t$ ) is continuous but that at the points $C_{1}, C_{2}$, etc. it has a discontinuous tangent. We see further that $S_{0}$ is the sum of two kinds of arcs, the arcs $A B_{1}, C_{1} B_{2}, C_{2} B_{3}$, etc. which are solutions of (1.3) and might be called regular ares, and the $\operatorname{arcs} B_{1} C_{1}, B_{2} C_{2}$, etc. which are solutions of (2.2) lying in planes $t=\tau_{j}$ and which might be called jump arcs since the arcs are traversed in a zero elapse of $t$. As a function of $t$ the solution is discontinuous and jumps from $B_{j}$ to $C_{j}$ at $t=\tau_{j}, j=1,2, \ldots, N$.

The condition (2.1) can be weakened by allowing $I$ to vanish on $g=0$ but requiring that $I$ be different from zero and of the same sign off $g=0$ in the neighborhood of $B$. A somewhat simpler situation where there will be no jump at all arises when $I$ changes sign in passing through $g=0$. These cases will not be pursued further.

In what follows the norm of a vector is defined as $|x|=\Sigma\left|x_{i}\right|$.

The basic results for (1.2) are given in the following theorems, 
Theorem 1. Let the degenerate system (1.3) have a solution $S_{0}$ in the sense defined above, for $\alpha \leqq t \leqq \beta$. Let $\varepsilon>0$. If $\varepsilon, \delta_{1}$ and $\delta_{2}$ are small enough there is a solution $x(t), u(t)$ of $(1.2)$ over $(\alpha, \beta)$ for any set of initial values satisfying

$$
\begin{gathered}
|x(\alpha)-y(\alpha)|+|u(\alpha)-v(\alpha)| \leqq \delta_{1} \\
\left|\frac{d u(\alpha)}{d t}-\frac{d v(\alpha)}{d t}\right| \leqq \frac{\delta_{2}}{\varepsilon}
\end{gathered}
$$

Moreover as $\varepsilon, \delta_{1}$ and $\delta_{2}$ tend to zero, the curve representing the solution $x(t), u(t)$ in $(x, u, t)$ tends to $S_{0}$. In particular for any fixed $\delta>0$

$$
|x(t)-y(t)|+|u(t)-v(t)|
$$

tends uniformly to zero over the intervals, $\alpha \leqq t \leqq \tau_{1}-\delta, \tau_{1}+\delta \leqq t \leqq \tau_{2}-\delta, \ldots$, $\tau_{N}+\delta \leqq t \leqq \beta$, as $\varepsilon, \delta_{1}$ and $\delta_{2} \rightarrow 0$. Also

$$
\frac{d u}{d t}-\frac{d v}{d t}
$$

tends uniformly to zero over the intervals $\alpha+\delta \leqq t \leqq \tau_{1}-\delta, \tau_{1}+\delta \leqq t \leqq \tau_{2}-\delta, \ldots$, $\tau_{N}+\delta \leqq t \leqq \beta$ as $\varepsilon, \delta_{1}$ and $\delta_{2} \rightarrow 0$. The same is true for $\frac{d^{2} u}{d t^{2}}-\frac{d^{2} v}{d t^{2}}$.

Theorem 2. It is also the case that if $S_{0}$ is a solution of (1.3) for $\alpha \leqq t \leqq \beta$ then corresponding to any set of initial values sufficiently near $y(\alpha), v(\alpha)$ there is a solution of (1.3) which tends to $S_{0}$ as the initial values tend to those of $S_{0}$. Moreover the convergence is uniform in $t$ if the portions of $S_{0}$ between $\tau_{j} \pm \delta$ are omitted.

Theorem 2 is in a sense Theorem 1 for the case $\varepsilon=+0$.

In what follows let us denote by $\frac{\partial}{\partial a}$ differentiation with respect to one of the $n+1$ initial coordinates $(x(\alpha), u(\alpha))$ or with respect to the corresponding initial coordinate of $(y(\alpha), v(\alpha))$. Then we have

Theorem 3. Subject to the same hypothesis as Theorem 1 we have

$$
\begin{gathered}
\left|\frac{\partial x(t)}{\partial a}-\frac{\partial y(t)}{\partial a}\right|+\left|\frac{\partial u(t)}{\partial a}-\frac{\partial v(t)}{\partial a}\right| \rightarrow 0, \\
\left|\frac{\partial}{\partial a} \frac{d u}{d t}-\frac{\partial}{\partial a} \frac{d v}{d t}\right| \rightarrow 0
\end{gathered}
$$

for $\varepsilon, \delta_{1}$, and $\delta_{2} \rightarrow 0$, the convergence being uniform for (2.8) and (2.9) over the same 
intervals of $t$ as for (2.6) and (2.7) respectively. Moreover denoting the initial value of $d u / d t$ at $\alpha$ by $b$ we have

and

$$
\left|\frac{\partial x(t)}{\partial b}\right|+\left|\frac{\partial u(t)}{\partial b}\right| \rightarrow 0
$$

$$
\left|\frac{\partial}{\partial b} \frac{d u}{d t}\right| \rightarrow 0
$$

uniformly over the same sets of intervals as for (2.6) and (2.7) respectively as $\varepsilon, \delta_{1}$ and $\delta_{2} \rightarrow 0$.

Theorem 4. The functions $\frac{\partial y(t)}{\partial a}$ and $\frac{\partial v(t)}{\partial a}$ are uniformly continuous with respect to changes in the initial values of $y$ and $v$ at $\alpha$ over the same set of intervals of $t$ as in Theorem 2.

Theorem 4 is in a sense Theorem 3 for the case $\varepsilon=+0$.

As an application of these theorems in case the right members do not contain $t$ or in case they are periodic in $t$ we have the fact that if the degenerate system (1.3) has a periodic solution and if the Jacobian associated with the determination of this solution by varying initial coordinates is different from zero, then it follows by Theorem 3 that (1.2) will also have a periodic solution. We shall show this in $\S 8$.

3. We shall require several lemmas. The first is well known.

Lemma 1. Let $\zeta(t)$ be a vector with an integrable derivative and let

$$
\left|\frac{d \zeta}{d t}\right| \leqq a(t)+b(t)|\zeta|, \quad t_{\mathbf{0}} \leqq t \leqq t_{\mathbf{1}},
$$

where $b(t) \geqq 0$. Then for $t_{0} \leqq t \leqq t_{1}$,

$$
\left|\zeta(t)-\zeta\left(t_{0}\right)\right| \leqq\left|\zeta\left(t_{0}\right)\right|\left(e^{\int t_{t_{0}}^{t} b(s) d s}-1\right)+\int_{t_{0}}^{t} a(\tau) e^{\int_{\tau}^{t} b(s) d s} d \tau
$$

Clearly (3.1) implies

$$
|\zeta(t)| \leqq\left|\zeta\left(t_{0}\right)\right| e^{\int_{t_{0}}^{t} b(s) d s}+\int_{t_{0}}^{t} a(\tau) e^{\int_{\tau}^{t} b(s) d s} d \tau
$$

Corresponding results hold if $t_{1}<t_{0}$. 
Proof. Let

$$
Z(t)=\int_{t_{10}}^{t}\left|\frac{d \zeta}{d t}\right| d t
$$

Then $\left|\zeta(t)-\zeta\left(t_{0}\right)\right| \leqq Z(t)$ and (3.0) becomes

Thus

$$
\frac{d Z}{d t} \leqq a(t)+b(t) Z+b(t)\left|\zeta\left(t_{0}\right)\right|
$$

$$
\left(\frac{d Z}{d t}-b(t) Z\right) e^{-\int_{\iota_{0}}^{t} b(s, d s} \leqq\left(a(t)+b(t)\left|\zeta\left(t_{0}\right)\right|\right) e^{-\int_{t_{0}}^{t} b(s) d s} .
$$

Thus integrating we find

$$
Z(t) e^{-\int_{t_{0}}^{t} b(s) d s} \leqq \int_{t_{0}}^{t}\left(a(\tau)+b(\tau)\left|\zeta\left(t_{0}\right)\right|\right) e^{-\int_{t_{0}}^{\tau} \tau_{(s) d s}} d \tau
$$

from which (3.1) follows.

The next lemma is very similar to one Friedrichs and Wasow [2]. Here $z$ is a vector and $w$ is a scalar and $L(z, w, t)$ and $M(z, w, t)$ are vectors while $H(z, w, t)$ and $J(z, w, t)$ are scalars.

Lemma 2. Let $z(t), w(t)$ satisfy

$$
\frac{d z}{d t}=L \frac{d w}{d t}+M, \quad \varepsilon \frac{d^{2} w}{d t^{2}}+H \frac{d w}{d t}=J
$$

for $\alpha \leqq t \leqq \gamma$ where $L, M, H$ and $J$ are continuous in the region given by $\alpha \leqq t \leqq \gamma$ and $|z|+|w| \leqq \lambda$ for some $\lambda>0$. In this region let

$$
|L| \leqq k,|M| \leqq k(|z|+|w|)+\varepsilon_{1},|J| \leqq k(|z|+|w|)+\varepsilon_{1}, H \geqq m>0 .
$$

Moreover at $t=\alpha$ let

$$
|z(\alpha)|+|w(\alpha)| \leqq \delta_{1}, \quad\left|\frac{d w(\alpha)}{d t}\right| \leqq \frac{\delta_{2}}{\varepsilon} .
$$

Let $k_{1}=k(k+m+1) / m$ and let

$$
\Delta=\frac{4(k+1)^{2}(m+1)}{m k}\left(\delta_{1}+\delta_{2}+\varepsilon_{1}\right) .
$$

Then for $\alpha \leqq i \leqq \gamma$, and if $\varepsilon_{1}, \delta_{1}$, and $\delta_{2}$ are small enough

and

$$
|z(t)|+|w(t)| \leqq \Delta e^{k_{1}(t-x)}
$$




$$
\left|\frac{d w}{d t}\right| \leqq \frac{\delta_{2}}{\varepsilon} e^{-m(t-\alpha) / \varepsilon}+\frac{k \Delta}{m} e^{k_{1}(t-\alpha)} .
$$

Proof of Lemma 2. From (3.3) we have if we set $\frac{d w}{d t}=\theta$,

$$
\frac{d z}{d t}=L \theta+M, \quad \frac{d w}{d t}=\theta, \quad \varepsilon \frac{d \theta}{d t}+H \theta=J .
$$

Or

$$
\left|\frac{d z}{d t}\right|+\left|\frac{d w}{d t}\right| \leqq(k+1)|\theta|+k(|z|+|w|)+\varepsilon_{1} .
$$

If $\zeta$ denotes the vector $(z, w)$ then Lemma 1 yields

$$
|z(t)|+|w(t)| \leqq\left(\frac{\varepsilon_{1}}{k}+\delta_{1}\right) e^{k(t-\alpha)}+(k+1) \int_{\alpha}^{t}|\theta(\tau)| e^{k(t-\tau)} d \tau .
$$

From the last equation of (3.6)

Or since $H \geqq m>0$

$$
\theta e^{\frac{1}{\varepsilon} \int_{\alpha}^{t} H(s) d s}=\theta(\alpha)+\frac{1}{\varepsilon} \int_{\alpha}^{t} J e^{\frac{1}{\varepsilon} \int_{\alpha}^{\tau} H(s) d s} d \tau .
$$

Thus

$$
|\theta(t)| \leqq|\theta(\alpha)| e^{-m(t-\alpha) / \varepsilon}+\frac{1}{\varepsilon} \int_{\alpha}^{t}|J| e^{-m(t-\tau) / \varepsilon} d \tau .
$$

From (3.7)

$$
|\theta(t)| \leqq \frac{\delta_{2}}{\varepsilon} e^{-m(t-\alpha) / \varepsilon}+\frac{k}{\varepsilon} \int_{\alpha}^{t}(|z(\tau)|+|w(\tau)|) e^{-m(t-\tau) / \varepsilon} d \tau+\frac{\varepsilon_{1}}{m}
$$

$$
|\theta(t)| \leqq \frac{\delta_{2}}{\varepsilon} e^{-m(t-\alpha) / \varepsilon}+\frac{\varepsilon_{1}}{m}+\frac{k}{m} e^{k(t-\alpha)}\left(\frac{\varepsilon_{1}}{k}+\delta_{1}\right)+\frac{k(k+1)}{\varepsilon} \int_{\alpha}^{t} e^{-m(t-\tau) / \varepsilon} d \tau \int_{\alpha}^{\tau}|\theta(\sigma)| e^{k(\tau-\sigma)} d \sigma .
$$

From this

(3.9) $|\theta(t)| \leqq \frac{\delta_{2}}{\varepsilon} e^{-m(t-\alpha) / \varepsilon}+\frac{k}{m} e^{k(t-\alpha)}\left(\frac{2 \varepsilon_{1}}{k}+\delta_{1}\right)+\frac{k(k+1)}{m} e^{k(t-\alpha)} \int_{\alpha}^{t}|\theta(\sigma)| e^{-k(\sigma-\alpha)} d \sigma$.

Applying Lemma 1 with $\zeta=\int_{\alpha}^{t}|\theta(\sigma)| e^{-k(\sigma-\alpha)} d \sigma$ we get

$$
\int_{\alpha}^{t}|\theta(\sigma)| e^{-k \cdot \sigma-\alpha)} d \sigma \leqq e^{k(k+1)(t-\alpha) / m}\left(\delta_{1}+\frac{\delta_{2}}{m}+\frac{2 \varepsilon_{1}}{k}\right) .
$$

In (3.7) and (3.9) this proves the lemma providing $\varepsilon_{1}, \delta_{1}$ and $\delta_{2}$ are small enough so that $\Delta e^{k_{1}(\gamma-\alpha)}<\lambda$. 
Before turning to the proof of Theorem 1 we make the following observation which is valid for Theorem 1,2,3 and 4. It suffices to take the case where there is only one jump arc on $S_{0}$ since by repeated use of the theorem for the case of one jump arc, the theorem for $N$ jump ares follows at once. Therefore we shall assume that $S_{0}$ is of the form $A B_{1} C_{1} A^{\prime}$. Clearly there will be no confusion if we call $S_{0}, A B C A^{\prime}$.

The proof of Theorem 1 is divided into four parts. In the first part we proceed from $A$ to a point short of $B$; the second part involves the immediate vicinity of $B$; the third part takes the arc $B C$ with the two small portions at ends $B$ and $C$ omitted; the fourth part takes the rest of $S_{0}$ to $A^{\prime}$. We shall use $K$ throughout the paper to represent finite constants which depend on the bounds of $|f|,|\varphi|,|g|,|h|$ and their first order partial derivatives in $R$ or part of $R$ for small $\varepsilon$, on the distance from $S_{0}$ to the nearest point on the boundary of $R$, and on the length of $S_{0}$. In particular the constants $K$ will remain finite as $\varepsilon+\delta_{1}+\delta_{2} \rightarrow+0$. Any deviation from this use of $K$ will be noted.

\section{Proof of Theorem 1, Part 1.}

Here we prove Theorem 1 for the interval $\alpha \leqq t \leqq \gamma$ where $\gamma<\tau_{1}$. If we denote $x-y$ by $z$ and $u-v$ by $w$ we have from (1.2) and (1.3)

$$
\begin{gathered}
\frac{d z}{d t}=f(x, u, t, \varepsilon) \frac{d w}{d t}+F_{1} \\
\varepsilon \frac{d^{2} w}{d t^{2}}+g(x, u, t, \varepsilon) \frac{d w}{d t}=F_{2}
\end{gathered}
$$

where $F_{1}$ is a vector and

and

$$
F_{1}=-[f(x, u, t, \varepsilon)-f(y, v, t, 0)] \frac{h(y, v, t, 0)}{g(y, v, t, 0)}+\varphi(x, u, t, \varepsilon)-\varphi(y, v, t, 0)
$$

$$
\begin{aligned}
F_{2} & =[g(x, u, t, \varepsilon)-g(y, v, t, 0)] \frac{h(y, v, t, 0)}{g(y, v, t, 0)}-h(x, u, t, \varepsilon) \\
& +h(y, v, t, 0)+\frac{\varepsilon}{g^{2}}\left[\left[g(y, v, t, 0) \frac{d h}{d t}-h(y, v, t, 0) \frac{d g}{d t}\right]\right.
\end{aligned}
$$

Let the minimum of $g(y(t), v(t), t, 0)$ over $(\alpha, \gamma)$ be denoted by $2 m$ unless this minimum exceeds 1 in which case we take $m=1$. Then so long as $(x, u, t)$ is in $R$ we have using the mean value theorem,

$$
\left|F_{1}\right| \leqq \frac{K}{m}(|z|+|w|+|f(y, v, t, \varepsilon)-f(y, v, t, 0)|+|\varphi(y, v, t, \varepsilon)-\varphi(y, v, t, 0)|) .
$$


Since $f$ and $\varphi$ are uniformly continuous for small $\varepsilon$ there must be a continuous function $\psi(\varepsilon)$ such that $\psi(0)=0$ and such that

$$
|f(y, v, t, \varepsilon)-f(y, v, t, 0)| \leqq \psi(\varepsilon)
$$

with similar results for $\varphi, g$ and $h$ so long as $(y, v, t)$ is in $R$.

Clearly we can choose $\psi(\varepsilon)>\varepsilon$. We get

$$
\left|F_{1}\right| \leqq \frac{K}{m}(|z|+|w|+\psi(\varepsilon))
$$

and similarly computing $\frac{d h}{d t}$ and $\frac{d g}{d t}$ we find

$$
\left|\boldsymbol{F}_{2}\right| \leqq \frac{K}{m^{3}}(|z|+|w|+\psi(\varepsilon)) .
$$

We have $f(x, u, t, \varepsilon)=f(y(t)+z, v(t)+w, t, \varepsilon)$ and similarly for $\varphi, g$, and $h$. If we now apply Lemma 2 to (3.10) and (3.11) and make use of (3.12) and (3.13) we see that if $\varepsilon, \delta_{1}$, and $\delta_{2}$ as defined in the statement of Theorem 1 are small enough, $(x, u, t)$ is in $R$ for $\alpha \leqq t \leqq \gamma$, and indeed for $\alpha \leqq t \leqq \gamma$, at least so long as $g(x, u, t, \varepsilon) \geqq m$, and

$$
|x(t)-y(t)|+|u(t)-v(t)| \leqq e^{K / m^{4}}\left(\delta_{1}+\delta_{2}+\psi(\varepsilon)\right)
$$

$$
\left|\frac{d u}{d t}-\frac{d v}{d t}\right| \leqq \frac{\delta_{2}}{\varepsilon} e^{-m(t-\alpha) / \varepsilon}+e^{K / m^{4}}\left(\delta_{1}+\delta_{2}+\psi(\varepsilon)\right) .
$$

From (3.14) and the continuity of $g$ we see that we will indeed have $g(x, u, t, \varepsilon) \geqq m$ if $\varepsilon, \delta_{1}$ and $\delta_{2}$ are small enough. We see from (3.14) and (3.15) that for $t<\tau_{1}$, Theorem 1 is established except for the difference of the second derivatives of $u$ and $v$. This we shall show in Lemma 4 . The discontinuity at $\tau_{1}$ is precisely the point of interest here and we begin to handle it in $\S 4$.

4. In the next part of the proof of Theorem 1 we shall show that for small $\varepsilon, \delta_{1}$ and $\delta_{2}$ the solution of (1.2) intersects the hypersurface $g=0$ at a point which tends to $B$ as $\varepsilon, \delta_{1}$ and $\delta_{2} \rightarrow 0$. Moreover as $\varepsilon, \delta_{1}, \delta_{2} \rightarrow 0, \frac{d u}{d t} \rightarrow \infty$ or $-\infty$ at the point of intersection.

\section{Proof of Theorem 1, Part 2.}

We shall consider here the case where $h(y, v, t, 0)<0$ at $B$. The case where $h>0$ is treated in exactly the same manner. At $B$ we have $t=\tau_{1}$ and we shall designate $y$ at $B$ by $y_{B}$ and $v$ by $v_{B}$. As $t \rightarrow \tau_{1}-0$ we have, since $h<0, \frac{d v}{d t}=$

6. Acta mathematica, 82. Imprimé le 18 decembre 1949. 
$-\frac{h}{g} \rightarrow+\infty$. Let $v_{1}<v_{B}$ be near enough to $v_{B}$ so that as $t$ increases from $\alpha$ toward $\tau_{1}$ there is a value of $t=t_{1}$ near to $\tau_{1}$ such that $v\left(t_{1}\right)=v_{1}$ and $d v / d t$ is large for $t_{1} \leqq t<\tau_{1}$. We shall denote the point $\left(y\left(t_{1}\right), v\left(t_{1}\right), t_{1}\right)$ by the letter $Q$. If we choose $\gamma$ so that $t_{1}<\gamma<\tau_{1}$, and apply the results (3.14) and (3.15) we see that if $\varepsilon, \delta_{1}$ and $\delta_{2}$ are small enough then for some $t$ (which tends to $t_{1}$ as $\varepsilon, \delta_{1}, \delta_{2} \rightarrow 0$ ) we have $u(t)=v_{1}$. Let us denote this point by $P$. Then at $P$ we have $t=t_{P}, x\left(t_{P}\right)=x_{P}$ and $u=u_{P}=u\left(t_{P}\right)=v_{1}$. Also as $\varepsilon, \delta_{1}, \delta_{2} \rightarrow 0, P \rightarrow Q$. Clearly we can choose $Q$ as near to $B$ as we wish.

We now change from $t$ to $v$ (and $u$ ) as the independent variable. Since when $v=u$ the values of $t$ for the points on $S_{0}$ and the solution of (1.2) are not in general equal we will reserve $t$ for the system (1.2) and in this section designate the variable $t$ for (1.3) by the letter $s$. We have then that (1.2) can be written as

$$
\frac{d x}{d u}=f+\varphi p, p=\frac{d t}{d u}, \varepsilon \frac{d p}{d u}=p^{2} g+p^{3} h
$$

where $f=f(x, u, t, \varepsilon)$, etc. while (1.3) becomes

$$
\frac{d y}{d v}=f+\varphi q, q=\frac{d s}{d v}, \quad 0=g+q h .
$$

where $f=f(y, v, s, 0)$, etc. Since $u$ and $v$ are the independent variables here we can with no confusion use them interchangeably. We consider (4.0) for $u \geqq v_{1}$. The solution of (1.2) can now be regarded at least in certain range of $u$ as a solution of $(4.0)$.

Suppose $v_{2}>v_{B}$. Let us choose

$$
K_{1}>2(|f|+|\varphi|+1)
$$

for all $(x, u, t)$ in $R$. (Clearly $K_{1}$ is a $\left.K\right)$. Let $R_{1}$ denote the region of $(x, u, t)$ bounded by the planes $u=v_{1}$, and $u=v_{2}$ and by

$$
\left|x-x_{P}\right|+\left|t-t_{P}\right| \leqq K_{1}\left(v_{2}-v_{1}\right)
$$

Clearly if $v_{1}$ and $v_{2}$ are chosen near enough to $v_{B}$ we have $R_{1}$ contained in $R$ for small $\varepsilon$. If we consider the change in $g$ as we follow. a solution of (4.0) we have

Or

$$
\frac{d g}{d u}=\sum \frac{\partial g}{\partial x_{i}} \frac{d x_{i}}{d u}+\frac{\partial g}{\partial u}+\frac{\partial g}{\partial t} \frac{d t}{d u}
$$




$$
\frac{d g}{d u}=J_{1}(x, u, t, \varepsilon)+p J_{2}(x, u, t, \varepsilon)
$$

where

$$
J_{1}=\sum \frac{\partial g}{\partial x_{i}} f_{i}+\frac{\partial g}{\partial u}, \quad J_{2}=\sum \frac{\partial g}{\partial x_{i}} \phi_{i}+\frac{\partial g}{\partial t} .
$$

We recall the definition of $I$ in (2.1) and our assumption that $I \neq 0$. Since $g(y(v), v, s(v), 0)>0$ for $v<v_{B}$ and zero at $v=v_{B}$ we see that $d g / d v \leqq 0$. This fact, the fact that $I \neq 0$ and the fact that $d v / d t$ is large implies that $I<0$. Let $K_{2}$ be chosen so that in $R$ and for small $\varepsilon,\left|J_{2}\right| \leqq K_{2}$. If $R_{1}$ is small enough, that is if $v_{1}$ and $v_{2}$ are near enough to $v_{B}$, we certainly have $J_{1}<\frac{1}{2} I$ and $h<\frac{1}{2} h_{B}$ for $(x, u, t)$ in $R_{1}$ and for small $\varepsilon$.

Let $v_{1}$ be near enough to $v_{B}$ so that

$$
q\left(v_{\mathbf{1}}\right)=-\frac{g_{Q}}{h_{Q}}<-\frac{I}{10 K_{2}}
$$

This is possible since $g_{Q} \rightarrow 0$ as $v_{1} \rightarrow v_{B}$. Let $\varepsilon, \delta_{1}$ and $\delta_{2}$ be so small that $P$ is near enough to $Q$ and $p$ near enough to $q$ at $u=v_{1}$ so that

$$
0<p\left(v_{1}\right)<-\frac{2 g_{P}}{h_{B}}<-\frac{I}{4 K_{2}} .
$$

Now let us suppose that for our solution of (4.0) there is a $v_{3}, v_{1}<v_{3}<v_{2}$ such that for $v_{1} \leqq u<v_{3}, p(u)<-2 g_{P} / h_{B}$ but that for $u=v_{3}$ we have either $p=$ $-2 g_{P} / h_{B}$ or $(x, u, t)$ reaches the boundary of $R_{1}$. We shall show that this is impossible. By integrating

$$
\varepsilon \frac{d p}{p^{2}}=(g+p h) d u
$$

from $v_{1}$ to $v_{3}$ we see that $p>0$ since if $p=0$ the left side diverges. For $v_{1} \leqq u<v_{3}$, since $0<p<-2 g_{P} / h_{B}$, we can assume $p<1$ since we can take $Q$ and therefore $P$ near $B$ where $g$ vanishes. From (4.0)

$$
\left|x-x_{P}\right|+\left|t-t_{P}\right| \leqq \int_{v_{1}}^{v_{3}}(|f|+|\varphi|+1) d u<K_{1}\left(v_{2}-v_{1}\right) .
$$

Thus $(x(u), t(u))$ is in $R_{1}$ for $v_{1} \leqq u \leqq v_{3}$ and therefore we must have $p=-2 g_{P} / h_{B}$ at $u=v_{3}$. Since $p<-2 g_{P} / h_{B}<-I / 4 K_{2}$ for $v_{1} \leqq u<v_{3}$, we have

$$
\frac{d g}{d u}=J_{1}+p J_{2}<\frac{1}{2} I+p K_{2} \leqq \frac{1}{2} I-\frac{1}{4} I=\frac{1}{4} I<0 .
$$


Thus $g$ is decreasing as $u$ increases up to $v_{3}$. Since $g$ is decreasing we have at $v_{3}$

$$
g+p h<g_{P}+\frac{1}{2} p h_{B} \leqq g_{P}-g_{P}=0 .
$$

Thus by (4.3), $d p / d u<0$ at $v_{3}$. That is $p$ is decreasing and therefore we cannot have $p=-2 g_{P} / h_{B}$ for the first time at $v_{3}$.

We see then that our solution of (4.0) remains in $R_{1}$ and can be extended to $u=v_{2}$ and that $0<p<-2 g_{P} / h_{B}$ for $v_{1} \leqq u \leqq v_{2}$. We can take $v_{1}$ as close to $v_{B}$ as we wish. Thus $Q$ can be as near $B$ as we wish. By taking $\varepsilon, \delta_{1}$ and $\delta_{2}$ small enough we can bring $P$ as close to $Q$, and therefore to $B$, as we wish. The nearer we take $P$ to $B$ the smaller is $g_{P}$ and therefore the smaller is $p$ for $v_{1} \leqq u \leqq v_{2}$. Since $q=-g / h$ until $g=0$ after which $q$ is identically zero on $S_{0}$, until $v=v_{C}$, we see that $q$ for $v_{1} \leqq v \leqq v_{2}$ also gets smaller as we take $v_{1}$ nearer to $v_{B}$. Since $u$ and $v$ are independent variables for the respective systems we can identify $u$ with $v$. Thus we can write our differential equations as

$$
\frac{d x}{d v}=f(x, v, t, 0)+\omega_{1}, \quad \frac{d t}{d v}=p
$$

$$
\frac{d y}{d v}=f(y, v, s, 0)+\omega_{2}, \quad \frac{d s}{d v}=q,
$$

where $\omega_{1}=f(x, v, t, \varepsilon)-f(x, v, t, 0)+p \varphi(x, v, t, \varepsilon)$ and $\omega_{2}=q \varphi(y, v, s, 0)$. Using the facts just enumerated we have, for $v_{1} \leqq v \leqq v_{2}$

$$
\left|\omega_{1}\right|+\left|\omega_{2}\right| \leqq K\left(g_{P}+g_{Q}+\psi(\varepsilon)\right)
$$

where $\psi(\varepsilon)$ has the same properties as in $\S 3$. Applying a standard theorem to (4.5) relating two approximate solutions of a system or else setting $\zeta=(x-y, t-s)$ and making use of Lemma 1 we have

$$
|x(v)-y(v)|+|t(v)-s(v)| \leqq K\left[\psi(\varepsilon)+g_{P}+g_{Q}+\left|x\left(v_{1}\right)-y\left(v_{1}\right)\right|+\left|t\left(v_{1}\right)-s\left(v_{1}\right)\right|\right]
$$

for $v_{1} \leqq v \leqq v_{2}$. Since the right member can be made as small as we wish we have demonstrated Theorem 1 up to the intersection of the hyper-plane $v=v_{2}$ with $S_{0}$. We observe that $v_{2}$ can be kept fixed in the latter part of our argument as $\varepsilon, \delta_{1}$, and $\delta_{2} \rightarrow 0$ while $v_{1}$ must approach $v_{B}$.

Proof of Theorem 1, Part 3. Here we demonstrate Theorem 1 over the part of the jump arc $B C$ beginning with $v=v_{2}$ and ending short of the point $C$. 
Since $I<0$ and $g_{P}>0$ and is small, we see that (4.4) implies that the solution of (4.0) crosses the hypersurface $g=0$ in exactly one point which is near $B$. Let us designate the point where the solution crosses $g=0$ by the subscript 4 . We have by integrating (4.3)

$$
\varepsilon\left(\frac{1}{p_{4}}-\frac{1}{p}\right)=\int_{u_{4}}^{u}(g+p h) d u
$$

Or for $u \geqq u_{4}$ (and so long as $x$ and $p$ remains finite)

$$
\frac{\varepsilon}{p(u)}=\frac{\varepsilon}{p_{4}}-\int_{u_{4}}^{u} g d u-\int_{u_{4}}^{u} p h d u .
$$

In (4.6) $g$ is $g(x(u), u, t(u), \varepsilon)$ and similarly for $h$.

We have already seen that $p(u)<-2 g_{P} / h_{B}$ for $v_{1} \leqq v \leqq v_{2}$. Thus given any $\delta_{3}>0$, by taking $P$ near enough to $B$, we can make $p(u) \leqq \delta_{3}, v_{1} \leqq u \leqq v_{2}$. Given any $\delta_{4}>0$ we can choose $\varepsilon, \delta_{1}$, and $\delta_{2}$ small enough so that

$$
\left|x\left(v_{2}\right)-y\left(v_{2}\right)\right|+\left|t\left(v_{2}\right)-s\left(v_{2}\right)\right| \leqq \delta_{4} .
$$

Now so long as $p(u) \leqq \delta_{3}$ we have from comparing the two systems

$$
\begin{gathered}
\frac{d x}{d v}=f(x, v, t, \varepsilon)+p \varphi(x, v, t, \varepsilon), \frac{d t}{d v}=p \\
\frac{d y}{d v}=f(y, v, s, 0), \quad \frac{d s}{d v}=0
\end{gathered}
$$

for $v_{2} \leqq v<v_{C}$, just as in the argument following (4.5),

$$
|x-y|+|t-s| \leqq K\left(\delta_{3}+\delta_{4}+\psi(\varepsilon)\right) \text {. }
$$

We recall incidentally that on $B C, s(v)=\tau_{1}$. We shall show that $p(u) \leqq \delta_{3}$ almost up to $u=v_{C}$. We choose $\delta_{5}>0$ as small as we wish and then choose $\delta_{6}>0$ small enough so that

$$
\int_{v_{B}}^{v} g(y(v), v, s(v), 0) d v<-4 \delta_{6}, v_{2} \leqq v \leqq v_{C}-\delta_{5}
$$

This is possible since $g<0$ for $v_{B}<v \leqq v_{2}$ and since the continuous function of $v$

$$
\int_{v_{B}}^{v} g d v<0, v_{B}<v<v_{C}
$$

The logical procedure in this section is to first choose $\delta_{5}$ and then $\delta_{6}$. We then observe 
that we can require $\delta_{3}$ and $\delta_{4}$ to be as small as we wish if we make $\varepsilon, \delta_{1}$, and $\delta_{2}$ small enough. By taking $\delta_{3}, \delta_{4}$ and $\varepsilon$ small enough we have from (4.8) that for $v_{C}>v \geqq v_{2}$ and so long as $p \leqq \delta_{3}$

$$
|g(x(v), v, t(v), \varepsilon)-g(y(v), v, s(v), 0)|<\delta_{6} /\left(v_{C}-v_{B}+1\right) .
$$

We can also satisfy (4.10) for $v_{1} \leqq v \leqq v_{2}$ on the basis of Part 2 simply by taking $\varepsilon, \delta_{1}$, and $\delta_{2}$ small enough. Turning to (4.6) we have

$$
p(u)=\frac{\varepsilon}{\frac{\varepsilon}{p_{4}}-\int_{u_{4}}^{u} g(x(v), v, t(v), \varepsilon) d v-\int_{u_{4}}^{u} p h d v} .
$$

But by (4.10) followed by (4.9) we have for $v_{2}<u<v_{C}-\delta_{5}$

$$
\begin{gathered}
-\int_{u_{4}}^{u} g(x(v), v, t(v), \varepsilon) d v \geqq-\int_{u_{4}}^{u} g(y(v), v, s(v), 0) d v-\delta_{6} \\
\geqq 3 \delta_{6}-\left|\int_{v_{B}}^{u_{4}} g(y(v), v, s(v), 0) d v\right| .
\end{gathered}
$$

Since we can bring $u_{4}$ as close to $v_{B}$ as we wish by taking $\varepsilon, \delta_{1}$, and $\delta_{2}$ small enough, we can make

Thus

$$
\left|\int_{v_{B}}^{u_{4}} g d v\right|<\delta_{6}
$$

Also so long as $p \leqq \delta_{3}$,

$$
-\int_{u_{4}}^{u} g(x(v), v, t(v), \varepsilon) d v>2 \delta_{6}
$$

$$
\left|\int_{u_{1}}^{u} p h d v\right| \leqq K_{3} \delta_{3}
$$

If we choose $\delta_{3}$ small enough so $K_{3} \delta_{3}<\delta_{6}$ then certainly the last two inequalities used in (4.11) yield

$$
p(u)<\varepsilon / \delta_{6}
$$

up to the point where we first have either $p(u)=\delta_{3}$ or $u=v_{C}-\delta_{5}$. But if $\varepsilon$ is small enough so that $\varepsilon / \delta_{6}<\delta_{3}$ we certainly cannot have $p(u)=\delta_{3}$ in $u_{4} \leqq u \leqq v_{C}-\delta_{5}$. Thus (4.8) holds in this range and we have established Theorem 1 on $S_{0}$ up to any point just short of $C$.

Theorem 1, Part 4. Here we prove Theorem 1 in the neighborhood of $C$ and beyond. Given any $\delta_{5}>0$ we saw that for $v_{B} \leqq v \leqq v_{C}-\delta_{5}$ we have $|x-y|+\left|t-\tau_{1}\right|+$ 
$\frac{d t}{d u} \rightarrow 0$ as $\varepsilon, \delta_{1}, \delta_{2} \rightarrow 0$. We now show that in the neighborhood of $C, p$ grows quickly. As we have already seen from (4.3) $p>0$ so long as $p$ remains small. We shall show that $p$ must exceed $\min \left[g_{C} /(2 K), 1\right]$ in the neighborhood of $C$ where now $K$ is the $\max$ of $|h|$ in $R$.

Let us denote a value of $v<v_{C}$ by $v_{5}$ and a value $v>v_{C}$ by $v_{6}$. We choose $v_{5}$ and $v_{6}$ near $v_{C}$. So long as $p<1$ we can construct $R_{2}$, much like $R_{1}$, so that if $v_{5}$ and $v_{6}$ are chosen close enough together the solution of (4.0) can be continued up to $v_{6}$ and will lie in $R_{2}$ which in turn will lie in $R$. This is certainly the case then if $p$ is small. We can also choose $v_{5}$ and $v_{6}$ near enough to $v_{C}$ so that in $R_{2}, g>\frac{1}{2} g_{C}>0$.

Integrating (4.3) between $v_{1}$ and $u_{4}$ we have

$$
\frac{\varepsilon}{p_{4}}=\frac{\varepsilon}{p_{1}}-\int_{v_{1}}^{u_{4}}(g+p h) d u .
$$

Since we can choose $v_{1}$ as close to $v_{B}$ as we want and since as $\varepsilon, \delta_{1}$, and $\delta_{2} \rightarrow 0$, $u_{4} \rightarrow v_{B}$ we see that we can require that

for any $\delta_{7}>0$. Thus

$$
\left|\int_{v_{1}}^{u_{4}}(g+p h) d u\right|<\delta_{7}
$$

$$
\frac{\varepsilon}{p_{4}}<\frac{\varepsilon}{p_{1}}+\delta_{7}
$$

Now since we can choose $\varepsilon, \delta_{1}$, and $\delta_{2}$ as small as we wish after having selected $v_{1}$ we can make $\varepsilon / q_{1}$ and therefore also $\varepsilon / p_{1}$ as small as we want. Thus we can require

Thus (4.6) gives

$$
\frac{\varepsilon}{p_{4}}<2 \delta_{7}
$$

$$
p(u)>\frac{\varepsilon}{2 \delta_{7}-\int_{u_{4}}^{u} g d u-\int_{u_{4}}^{u} p h d u} .
$$

From the result of part 3 it follows that if we take $v_{\overline{0}}$ close enough to $v_{C}$ and $\varepsilon, \delta_{1}$, and $\delta_{2}$ small enough we have

$$
\left|\int_{u_{4}}^{v_{5}}(g+p h) d u\right|<\delta_{7} .
$$

We see that the choice of $\delta_{7}$ affects the choice of $v_{5}$ but not $v_{6}$. We have 


$$
p(u)>\frac{\varepsilon}{3 \delta_{7}-\int_{v_{5}}^{u}(g+p h) d u} .
$$

Now let us assume that

$$
g+p h \geqq \frac{5 \delta_{7}}{v_{6}-v_{5}}, \quad v_{5} \leqq u \leqq v_{6} .
$$

Then we find from (4.13) that $p(u) \rightarrow \infty$, contrary to our assumption that $p<1$. Thus (4.14) cannot hold and we have

$$
p h<\frac{5 \delta_{7}}{v_{6}-v_{5}}-g<\frac{5 \delta_{7}}{v_{6}-v_{C}}-g
$$

for some $u, v_{5}<u<v_{6}$. If $\delta_{7}$ is small enough this can be replaced by

$$
p h<-\frac{1}{2} g_{C} .
$$

Since $g_{C}>0$ we must have $h \neq 0$. If $h>0$ we have $p<0$ which is impossible. If $h<0$ and $|h|<K$ we have $p>g_{C} /(2 K)$. Thus if $p<1$ we certainly have $p>g_{C} /(2 K)$ for some $u, v_{5}<u<v_{8}$.

Since we can choose $v_{6}$ as close to $C$ as we wish we see that indeed we can enclose $C$ in a sphere in $(x, u, t)$ with center at $C$ and of radius arbitrarily small and that having chosen the sphere we can, by taking $\varepsilon, \delta_{1}$, and $\delta_{2}$ small enough be sure that $R_{2}$ lies in the sphere and that the solution of $(4.0)$ enters the sphere with $p$ very small but at some point in the sphere $p=\min \left(g_{C} /(2 K), 1\right)$. Let us denote this point by $D$. At $D$ we transform back to the original independent variables. To show that the solution (1.3) changes but little from $\tau_{1}$ to $t_{D}$, irrespective of whether $\tau_{1}-t_{D}$ is positive or negative we have only to note that $t_{D}-\tau_{1} \rightarrow 0$ as $\varepsilon, \delta_{1}, \delta_{2} \rightarrow 0$. We can now apply Lemma 2 at $D$ as we did at the point $A$ and we get Theorem 1 for the range of $t$ given by $\max \left(\tau_{1}, t_{D}\right) \leqq t \leqq \beta$.

The proof of Theorem 2 is very much simpler and shorter than that of Theorem 1. It is quite direct except near $B$ where we must use an argument similar to that used in the case of Theorem 1, Part. 2.

5. The proofs of Theorems 3 and 4 proceed along somewhat different lines. We shall first prove Theorem 4 . We use the letter $a$ to designate an initial value of $x_{i}$ and $y_{i}$ or of $u$ and $v$. The initial value of $\frac{d u(\alpha)}{d t}$ which has no counterpart in the degenerate system we shall denote by $b$. We observe that at $t=\alpha, \partial y_{i} / \partial a$ is zero 
unless $a$ is the intial value of $y_{i}$ in which case $\partial y_{i} / \partial a=1$ at $t=\alpha$. Similarly with $\partial v / \partial a$. We have $\frac{\partial y}{\partial a}=\frac{\partial x}{\partial a}$ and $\frac{\partial v}{\partial a}=\frac{\partial u}{\partial a}$ at $t=\alpha$ since $a$ represents the same initial coordinate in $(y, v)$ as in $(x, u)$. We always have $\frac{\partial}{\partial a}\left(\frac{d u}{d t}\right)=0$ at $t=\alpha$.

Proof of Theorem 4. We have on differentiating with respect to $a$

$$
\begin{aligned}
& \frac{d}{d t} \frac{\partial y}{\partial a}=f \frac{d}{d t} \frac{\partial v}{\partial a}+\frac{\partial f}{\partial a} \frac{d v}{d t}+\frac{\partial \varphi}{\partial a} \\
& \frac{d}{d t} \frac{\partial v}{\partial a}=\frac{h \frac{\partial g}{\partial a}-g \frac{\partial h}{\partial a}}{g^{2}}
\end{aligned}
$$

Here $f=f(y, v, t, 0)$, etc. and

$$
\frac{\partial f}{\partial a}=\sum \frac{\partial f}{\partial y_{i}} \frac{\partial y_{i}}{\partial a}+\frac{\partial f}{\partial v} \frac{\partial v}{\partial a}
$$

and similarly for $\varphi, g$ and $h$. The system (5.0) is linear in $\partial y / \partial a$ and $\partial v / \partial a$ and the coefficients are continuous for $\alpha \leqq t<\tau_{1}$. The initial values are known and thus $\partial y / \partial a$ and $\partial v / \partial a$ are determined for $\alpha \leqq t<\tau_{1}$. Moreover for $\alpha \leqq t \leqq \gamma<\tau_{1}$ the functions $\partial y / \partial a$ and $\partial v / \partial a$ are uniformly continuous with respect to $t$ and with respect to changes in the initial values of $y$ and $v$ at $t=\alpha$.

At $v=v_{0}<v_{B}$ but with $v_{0}$ near $v_{B}$ we change from $t$ to $v$ as the independent variable. We observe that $v=v_{0}$ determines a point on $S_{0}$ near $B$. As before it is convenient to replace $t$ by $s$ and reserve $t$ for the system (1.2). Let $s$ at $v=v_{0}$ be $s_{0}$ where clearly $s_{0}<\tau_{1}$. We denote $\partial y / \partial a$ and $\partial v / \partial a$ at $s=s_{0}$, or more precisely at $s=s_{0}-0$, by $\frac{\partial y\left(s_{0}\right)}{\partial a}$ and $\frac{\partial v\left(s_{0}\right)}{\partial a}$. We denote $\partial y / \partial a$ and $\partial s / \partial a$ at $v=v_{0}+0$ by $\frac{\partial y\left(v_{0}\right)}{\partial a}$ and $\frac{\partial s\left(v_{0}\right)}{\partial a}$. Clearly since $v\left(s_{0}\right)=v_{0}$ we have from $v(s, a)=v_{0}$,

Thus

$$
\frac{d v}{d s} \frac{\partial s\left(v_{0}\right)}{\partial a}+\frac{\partial v\left(s_{0}\right)}{\partial a}=0
$$

$$
\frac{\partial s\left(v_{0}\right)}{\partial a}=-\frac{\partial v\left(s_{0}\right)}{\partial a} \frac{d s\left(v_{0}\right)}{d v}
$$

Also 


$$
\frac{\partial y\left(v_{0}\right)}{\partial a}=\frac{d y}{d s} \frac{\partial s\left(v_{0}\right)}{\partial a}+\frac{\partial y\left(s_{0}\right)}{\partial a}=-\frac{d y\left(v_{0}\right)}{d v} \frac{\partial v\left(s_{0}\right)}{\partial a}+\frac{\partial y\left(s_{0}\right)}{\partial a} .
$$

For $v_{0} \leqq v \leqq v_{B}$ we have

$$
\frac{d}{d v} \frac{\partial y}{\partial a}=\frac{\partial f}{\partial a}+\frac{\partial \varphi}{\partial a} \frac{d s}{d v}+\varphi \frac{d}{d v} \frac{\partial s}{\partial a}, \quad \frac{d}{d v} \frac{\partial s}{\partial a}=\frac{g \frac{\partial h}{\partial a}-h \frac{\partial g}{\partial a}}{h^{2}}
$$

where the intitial values are taken from (5.1) and (5.2). For $v_{B} \leqq v \leqq v_{C}$ we have

$$
\frac{d}{d v} \frac{\partial y}{\partial a}=\frac{\partial f}{\partial a}, \quad \frac{d}{d v} \frac{\partial s}{\partial a}=0
$$

Thus $\partial y(v) / \partial a$ and $\partial s(v) / \partial a$ are determined up to the point $C$.

We have $g\left(y_{B}, v_{B}, \tau_{1}, 0\right)=0$. Thus

Since

$$
\sum \frac{\partial g}{\partial y_{i}} \frac{\partial y_{B i}}{\partial a}+\frac{\partial g}{\partial v} \frac{\partial v_{B}}{\partial a}+\frac{\partial g}{\partial t} \frac{\partial \tau_{1}}{\partial a}=0
$$

$$
\frac{\partial y_{B}}{\partial a}=\frac{d y}{d v} \frac{\partial v_{B}}{\partial a}+\frac{\partial y\left(v_{B}\right)}{\partial a}
$$

we have, recalling the definition of $I=I_{B}$,

$$
I \frac{\partial v_{B}}{\partial a}+\sum \frac{\partial g}{\partial y_{i}} \frac{\partial y_{i}\left(v_{B}\right)}{\partial a}+\frac{\partial g}{\partial t} \frac{\partial \tau_{1}}{\partial a}=0
$$

Since $I \neq 0$ we see that $\partial v_{B} / \partial a$ is determined in terms of $\partial \tau_{1} / \partial a$.

The coordinate $v_{C}$ is given by

$$
\int_{v_{B}}^{v_{C}} g\left(y(v), v, \tau_{1}, 0\right) d v=0 .
$$

Differentiating with respect to $a$ and recalling that $g=0$ at $B$ we have

We have

$$
g\left(y_{C}, v_{C}, \tau_{1}, 0\right) \frac{\partial v_{C}}{\partial a}+\int_{v_{B}}^{v_{C}}\left(\Sigma \frac{\partial g}{\partial y_{i}} \frac{\partial y_{i}}{\partial a}+\frac{\partial g}{\partial s} \frac{\partial \tau_{1}}{\partial a}\right) d v=0
$$

$$
\frac{\partial \tau_{1}}{\partial a}=\frac{\partial s\left(v_{B}\right)}{\partial a}+\frac{d s\left(v_{B}\right)}{d v} \frac{\partial v_{B}}{\partial a} .
$$

Since $d s\left(v_{B}\right) / d v=0$ we see ${ }^{1}$ that $\partial \tau_{1} / \partial a=\partial s\left(v_{B}\right) / \partial a$. Thus (5.5) determines $\partial v_{C} / \partial a$. Also

1 Strictly speaking we find $\partial v_{B} / \partial a$ in terms of $\partial \tau_{1} / \partial a$ and then $\partial \tau_{1} / \partial a$ in terms of $\partial v_{B} / \partial a$ which is not rigorous. Actually we should proceed with finite differences corresponding to an increment in $a, \Delta a$, and then take limits as $\Delta a \rightarrow 0$ to show the existence of $\partial v_{B} / \partial a$ from a single formula obtained by eliminating the term $\Delta \tau_{1} / \Delta a$ from the equation involving $I$ and the equation following (5.5). 


$$
\frac{\partial y_{C}}{\partial a}=\frac{d y}{d v} \frac{\partial v_{C}}{\partial a}+\frac{\partial y\left(v_{C}\right)}{\partial a}
$$

where $\partial y\left(v_{C}\right) / \partial a$ is $\partial y / \partial a$ at $v=v_{C}-0$. As we approach $C$ from $s>s_{C}$ we have

$$
\begin{aligned}
& \frac{\partial v_{C}}{\partial a}=\frac{\partial v\left(s_{C}\right)}{\partial a}+\frac{d v}{d s} \frac{\partial s_{C}}{\partial a} \\
& \frac{\partial y_{C}}{\partial a}=\frac{\partial y\left(s_{C}\right)}{\partial a}+\frac{d y}{d t} \frac{\partial s_{C}}{\partial a}
\end{aligned}
$$

where $\partial y\left(s_{C}\right) / \partial a$ is $\partial y(s) / \partial a$ at $s=s_{C}+0$ and similary for $\partial v\left(s_{C}\right) / \partial a$. However $\partial s_{C} / \partial a=$ $\partial \tau_{1} / \partial a$. Using (5.5) and (5.7) we have

$$
-\frac{1}{g_{c}} \int_{v_{B}}^{v_{C}}\left(\sum \frac{\partial g}{\partial y_{i}} \frac{\partial y_{i}}{\partial a}+\frac{\partial g}{\partial s} \frac{\partial \tau_{1}}{\partial a}\right) d v=\frac{\partial v\left(s_{C}\right)}{\partial a}+\frac{d v}{d s} \frac{\partial \tau_{1}}{\partial a}
$$

That is $\partial v\left(s_{C}\right) / \partial a$ is determined. Likewise $\partial y\left(s_{C}\right) / \partial a$ is determined. Indeed from (5.8) and (5.6)

$$
\frac{\partial y\left(s_{C}\right)}{\partial a}=\frac{d y\left(v_{C}\right)}{d v} \frac{\partial v_{C}}{\partial a}+\frac{\partial y\left(v_{C}\right)}{\partial a}-\frac{d y\left(s_{C}\right)}{d s} \frac{\partial \tau_{1}}{\partial a}
$$

From (5.7) and (1.3) therefore

$$
\frac{\partial y\left(s_{C}\right)}{\partial a}=f_{C} \frac{\partial v\left(s_{C}\right)}{\partial a}+\frac{\partial y\left(v_{C}\right)}{\partial a}-\varphi_{C} \frac{\partial \tau_{\mathbf{1}}}{\partial a}
$$

We now use (5.0) again from $s_{C} \leqq s \leqq \beta$ and thereby determine $\partial y(\beta) / \partial a$ and $\partial v(\beta) / \partial a$. The functions $\partial y(s) / \partial a$ and $\partial v(s) / \partial a$ are clearly uniformly continuous with respect to the initial values of $y$ and $v$ at $s=\alpha$ and with respect to $s$ for $\alpha \leqq s \leqq \tau_{1}-\delta$, $\tau_{1}+\delta \leqq t \leqq \beta$ for any given $\delta>0$.

This completes the proof of Theorem 4 .

6. We proceed now to the proof of Theorem 3. In the course of the proof we shall make use of Theorem 4 . We recall the remarks made at the beginning of $\S 5$ concerning the meaning of $a$ and $b$. We require the following result whieh is a slightly modified form of Lemma 2 and is proved in the same way.

Lemma 3. In Lemma 2 let $\varepsilon_{1}$ in (3.4) be replaced by

$$
\varepsilon_{1}+\frac{\varepsilon_{2}}{\varepsilon} e^{-m(t-\alpha) / \varepsilon}
$$


and let $\delta_{1}$ and $\delta_{2}$ be replaced by $\delta_{3}$ and $\delta_{4}$ respectively. Then the conclusion of Lemma 2 becomes

$$
\begin{gathered}
|z(t)|+|w(t)| \leqq \Delta_{1} e^{k_{1}(t-\alpha)} \\
\left|\frac{d w}{d t}\right| \leqq \frac{\delta_{4}}{\varepsilon} e^{-m(t-\alpha)^{\prime} \varepsilon}+\frac{\varepsilon_{2}(t-\alpha)}{\varepsilon^{2}} e^{-m(t-\alpha) \cdot \varepsilon}+\frac{k \Delta_{1}}{m} e^{k_{1}(t-\alpha)}
\end{gathered}
$$

for $\alpha \leqq t \leqq \gamma$ where $\Delta_{1}$ replaces $\triangle$ in Lemma 2 and

$$
\Delta_{1}=\frac{4(k+1)^{2}(m+1)^{2}}{k m^{2}}\left(\varepsilon_{1}+\varepsilon_{2}+\delta_{3}+\delta_{4}\right),
$$

and $k_{1}$ is the same as in Lemma 2 .

We begin by considering $\alpha \leqq t \leqq \gamma<\tau_{1}$.

Proof of Theorem 3, Part 1. Here we have

$$
\frac{d}{d t} \frac{\partial x}{\partial a}=f \frac{d}{d t} \frac{\partial u}{\partial a}+\frac{\partial f}{\partial a} \frac{d u}{d t}+\frac{\partial \varphi}{\partial a}
$$

$$
\varepsilon \frac{d^{2}}{d t^{2}} \frac{\partial u}{\partial a}+g \frac{d}{d t} \frac{\partial u}{\partial a}+\frac{\partial g}{\partial a} \frac{d u}{d t}+\frac{\partial h}{\partial a}=0
$$

where $f=f(x(t), u(t), t, \varepsilon)$, etc. and where

$$
\frac{\partial f}{\partial a}=\sum \frac{\partial f}{\partial x_{i}} \frac{\partial x_{i}}{\partial a}+\frac{\partial f}{\partial u} \frac{\partial u}{\partial a}
$$

etc. The system is linear in $\partial x / \partial a$ and $\partial u / \partial a$. The coefficients are all function of $t$ (and of $\varepsilon$ and the initial values of $x, u, d u / d t$ at $t=\alpha$ ).

For the degenerate system we have (5.0) where the last equation can be written as

$$
g \frac{d}{d t} \frac{\partial v}{\partial a}+\frac{\partial g}{\partial a} \frac{d v}{d t}+\frac{\partial h}{\partial a}=0 .
$$

Now let $z=\frac{\partial x}{\partial a}-\frac{\partial y}{\partial a}$ and $w=\frac{\partial u}{\partial a}-\frac{\partial v}{\partial a}$. The initial values of $z$ and $w$ are zero. From (6.0) and (5.0) we have

$$
\frac{d z}{d t}=f(x(t), u(t), t, \varepsilon) \frac{d w}{d t}+\left(\Sigma \frac{\partial f}{\partial x_{i}} z_{i}+\frac{\partial f}{\partial u} w\right) \frac{d v}{d t}+\sum \frac{\partial \varphi}{\partial x_{i}} z_{i}+\frac{\partial \varphi}{\partial u} w+F
$$

(6.2) $\frac{d^{2} w}{d t^{2}}+g(x(t), u(t), t, \varepsilon) \frac{d w}{d t}+\left(\sum \frac{\partial g}{\partial x_{i}} z_{i}+\frac{\partial g}{\partial u} w\right) \frac{d v}{d t}+\sum \frac{\partial h}{\partial x_{i}} z_{i}+\frac{\partial h}{\partial u} w+G=0$ 
where

$$
\begin{gathered}
F=\left(\frac{d u}{d t}-\frac{d v}{d t}\right)\left[\sum \frac{\partial f}{\partial x_{i}} z_{i}+\frac{\partial f}{\partial u} w+\sum \frac{\partial f}{\partial x_{i}} \frac{\partial y_{i}}{\partial a}+\frac{\partial f}{\partial u} \frac{\partial v}{\partial a}\right] \\
+\frac{d v}{d t}\left[\Sigma\left(\frac{\partial f}{\partial x_{i}}-\frac{\partial f}{\partial y_{i}}\right) \frac{\partial y_{i}}{\partial a}+\left(\frac{\partial f}{\partial u}-\frac{\partial f}{\partial v}\right) \frac{\partial v}{\partial a}\right] \\
+\Sigma\left(\frac{\partial \varphi}{\partial x_{i}}-\frac{\partial \varphi}{\partial y_{i}}\right) \frac{\partial y_{i}}{\partial a}+\left(\frac{\partial \varphi}{\partial u}-\frac{\partial \varphi}{\partial v}\right) \frac{\partial v}{\partial a}+\frac{d}{d t} \frac{\partial v}{\partial a}[f(x, u, t, \varepsilon)-f(y, v, t, 0)] .
\end{gathered}
$$

As $\varepsilon, \delta_{1}$, and $\delta_{2} \rightarrow 0$ we have for $\alpha \leqq t \leqq \gamma<\tau_{1}$ from Theorem 1, part 1, that

$$
\Sigma\left|\frac{\partial f(x, u, t, \varepsilon)}{\partial x_{i}}-\frac{\partial f(y, v, t, 0)}{\partial y_{i}}\right| \rightarrow 0
$$

uniformly where $x$ is $x(t), y$ is $y(t)$ etc. Similar results hold for the other differences that occur in $F$ except for the difference $\frac{d u}{d t}-\frac{d v}{d t}$. For the latter we have by (3.15)

$$
\left|\frac{d u}{d t}-\frac{d v}{d t}\right| \leqq \frac{\delta_{2}}{\varepsilon} e^{-m(t-\alpha) / \varepsilon}+e^{K / m^{4}}\left(\delta_{1}+\delta_{2}+\psi(\varepsilon)\right) .
$$

The term $\frac{d}{d t} \frac{\partial v}{\partial a}=\frac{\partial}{\partial a} \frac{d v}{d t}$ is replaced by use of $\frac{d v}{d t}=-\frac{h}{g}$. We have easily from (5.0) and Lemma 1 that the terms $\partial y_{i} / \partial a$ and $\partial v / \partial a$ are bounded over $\alpha \leqq t \leqq \gamma$, by a bound that depends only on $K$ and $m$. Thus so long as $|z|+|w| \leqq 1$ there is an $E$, a function of $K$ and $m$, which becomes large when $m$ gets small, such that

$$
|F| \leqq E\left[\psi\left(\varepsilon, \delta_{1}, \delta_{2}\right)+\frac{\delta_{2}}{\varepsilon} e^{-m(t-\alpha) ! \varepsilon}\right]
$$

where $\psi\left(\varepsilon, \delta_{1}, \delta_{2}\right)$ is a continuous function of $\varepsilon, \delta_{1}, \delta_{2}$, and $m$ which tends to zero when $\varepsilon, \delta_{1}$ and $\delta_{2} \rightarrow 0$. A similar result holds for $G$ which has terms like those of $F$ and the additional term $\frac{\partial d^{2} v}{\partial a} \frac{d t^{2}}{\text {. It }}$ is here that we use the existence of the second order partial derivatives of $g$ and $h$. We find that $G$ satisfies an inequality of the same form as (6.3). From the last equation of (5.0) $\frac{\partial}{\partial a} \frac{d v}{d t}$ at $t=\alpha$ can be computed. We have $\frac{\partial}{\partial a} \frac{d u}{d t}=0$ at $t=\alpha$. Thus $\frac{d w}{d t}$ at $t=\alpha$ is boundet and therefore $\delta_{4}$ is of the form $K \varepsilon$. With this we see that (6.1) and (6.2) satisfy the hypothesis of Lemma 3 where $\varepsilon_{1}=E \psi$ and $\varepsilon_{2}=E \delta_{2}$. Using Lemma 3 we see that Theorem 3 is valid for $\alpha \leqq t \leqq \gamma<\tau_{1}$ in so far as the derivatives with respect to $a$ are concerned. 
As regards the derivatives with respect to $b$ we use (6.0) without (5.0). We set $z=\partial x / \partial b$ and $w=\partial u / \partial b$ and apply Lemma 3. Since at $t=\alpha, z=w=0$ we have here that $\delta_{3}=0$. Since $\frac{\partial}{\partial b} \frac{d u}{d t}=1$ at $t=\alpha$ we have $\delta_{4}=\varepsilon$. Clearly for $|z|+|w| \leqq 1$ we have

$$
\begin{gathered}
\left|\frac{\partial f}{\partial b} \frac{d u}{d t}+\frac{\partial \varphi}{\partial b}\right| \leqq\left|\frac{\partial f}{\partial b}\right|\left|\frac{d u}{d t}-\frac{d v}{d t}\right|+\left|\frac{\partial f}{\partial b}\right|\left|\frac{d v}{d t}\right|+\left|\frac{\partial \varphi}{\partial b}\right| \\
\leqq E\left[\psi\left(\varepsilon, \delta_{1}, \delta_{2}\right)+\frac{\delta_{2}}{\varepsilon} e^{-m(t-\alpha) / \varepsilon}+|z|+|w|\right]
\end{gathered}
$$

where $E$ is the same kind of function as appeared in (6.3). A similar result holds for $\frac{\partial g}{\partial b} \frac{d u}{d t}+\frac{\partial h}{\partial b}$. If we now use Lemma 3 we find that $\left|\frac{\partial x(t)}{\partial b}\right|+\left|\frac{\partial u(t)}{\partial b}\right| \rightarrow 0$ uniformly over $\alpha \leqq t \leqq \gamma<\tau_{1}$ as $\varepsilon, \delta_{1}$ and $\delta_{2} \rightarrow 0$ while $\left|\frac{d}{d t} \frac{\partial u}{\partial b}\right| \rightarrow 0$ uniformly over $\alpha+\delta \leqq t \leqq$ $\gamma<\tau_{1}$ for any fixed $\delta>0$. This completes the proof of Theorem 3 over $\alpha \leqq t \leqq \gamma<\tau_{1}$.

\section{Theorem 3, Part 2.}

At $v=v_{0}<v_{B}$ we change from $t$ to $u$ (or $v$ ) as the independent variable. We have from (4.0)

$$
\begin{gathered}
\frac{d}{d u} \frac{\partial x}{\partial a}=\frac{\partial f}{\partial a}+\frac{\partial \varphi}{\partial a} p+\varphi \frac{\partial p}{\partial a}, \frac{d}{d u} \frac{\partial t}{\partial a}=\frac{\partial p}{\partial a} \\
\varepsilon \frac{d}{d u} \frac{\partial p}{\partial a}=2 p \frac{\partial p}{\partial a} g+p^{2} \frac{\partial g}{\partial a}+3 p^{2} \frac{\partial p}{\partial a} h+p^{3} \frac{\partial h}{\partial a}
\end{gathered}
$$

We also have (5.3) for the degenerate system. The initial values at $u=v=v_{0}$ of $\partial x\left(v_{0}\right) / \partial a$ and $\partial t\left(v_{0}\right) / \partial a$ may be found in the same way as $\partial y\left(v_{0}\right) / \partial a$ and $\partial s\left(v_{0}\right) / \partial a$ in $(5.1)$ and (5.2). Here $\partial x\left(v_{0}\right) / \partial a$ means $\partial x\left(v_{0}+0\right) / \partial a$ etc. From Part 1 we know that $\frac{\partial x\left(t_{0}\right)}{\partial a}-\frac{\partial y\left(s_{0}\right)}{\partial a}$ and $\frac{\partial u\left(t_{0}\right)}{\partial a}-\frac{\partial v\left(s_{0}\right)}{\partial a}$ can be made as small as we want by taking $\varepsilon, \delta_{1}$, and $\delta_{2}$ small enough. Thus $\frac{\partial x\left(v_{0}\right)}{\partial a}-\frac{\partial y\left(v_{0}\right)}{\partial a}$ and $\frac{\partial t\left(v_{0}\right)}{\partial a}-\frac{\partial s\left(v_{0}\right)}{\partial a}$ can be made as small as we wish.

We have also to consider $\partial p\left(v_{\mathbf{0}}\right) / \partial a$. We have since $p=1 / \frac{d u}{d t}$ 


$$
\frac{\partial p\left(v_{0}\right)}{\partial a}=-\frac{1}{\left(\frac{d u}{d t}\right)^{2}}\left[\frac{d^{2} u}{d t^{2}} \frac{\partial t\left(v_{0}\right)}{\partial a}+\frac{d}{d t} \frac{\partial u\left(t_{0}\right)}{\partial a}\right]
$$

A similar formula is valid for $\partial q\left(v_{0}\right) / \partial a$. The terms in (6.5) tend to the corresponding terms in $\partial q\left(v_{0}\right) / \partial a$, with the possible exception of $\frac{d^{2} u}{d t^{2}}$, as $\varepsilon, \delta_{1}$, and $\delta_{2} \rightarrow 0$ by Theorem 1 and Part 1 of Theorem 3. Here we have the following lemma.

Lemma 4. For $\alpha+\delta \leqq t \leqq \gamma<\tau_{1}$, where $\delta>0, \frac{d^{2} u}{d t^{2}}-\frac{d^{2} v}{d t^{2}} \rightarrow 0$ uniformlyas $\varepsilon, \delta_{1}$, and $\delta_{2} \rightarrow 0$ under the hypothesis of Theorem 1 . The result also holds for $\tau_{1}+\delta \leqq t \leqq \beta$.

We shall prove this lemma at the end of the section. We see now that indeed $\frac{\partial p\left(v_{0}\right)}{\partial a}-\frac{\partial q\left(v_{0}\right)}{\partial a} \rightarrow 0$ as $\varepsilon, \delta_{1}$, and $\delta_{2} \rightarrow 0$. It is easy to see that $\frac{\partial p(v)}{\partial a}-\frac{\partial q(v)}{\partial a} \rightarrow 0$ uniformly for $v_{0} \leqq v \leqq v_{B}-\delta$ for any $\delta>0$ as $\varepsilon, \delta_{1}$, and $\delta_{2} \rightarrow 0$. Since $q=\frac{d s}{d v}=-\frac{g}{h}$ we also see that $\frac{\partial q(v)}{\partial a}$ exists and is a continuous function of $v$ for $v<v_{B}$ and moreover $\frac{\partial q\left(v_{B}-0\right)}{\partial a}$ exists. If we now let $z$ represent $\left(\frac{\partial x(v)}{\partial a}-\frac{\partial y(v)}{\partial a}, \frac{\partial t(v)}{\partial a}-\frac{\partial s(v)}{\partial a}\right)$ then from (5.3) and (6.4)

$$
\begin{gathered}
\left|\frac{d z}{d v}\right| \leqq\left|\cdot \frac{\partial f(x, v, t, \varepsilon)}{\partial a}-\frac{\partial f(y, v, s, 0)}{\partial a}\right|+K\left|\frac{\partial p}{\partial a}-\frac{\partial q}{\partial a}\right| \\
\left|p \frac{\partial \varphi(x, v, t, \varepsilon)}{\partial a}-q \frac{\partial \varphi(y, v, s, 0)}{\partial a}\right|+\frac{\partial q}{\partial a} \mid \varphi(x, v, t, \varepsilon)-\varphi(y, v, s, 0 \mid
\end{gathered}
$$

So long as $|p|+|q| \leqq 1$ and as $|z| \leqq 1$,

$$
\begin{array}{r}
\left|\frac{d z}{d v}\right| \leqq K|z|+\Sigma\left|\frac{\partial f}{\partial x_{i}}-\frac{\partial f}{\partial y_{i}}\right|\left|\frac{\partial y_{i}}{\partial a}\right|+\left|\frac{\partial f}{\partial t}-\frac{\partial f}{\partial s}\right|\left|\frac{\partial s}{\partial a}\right| \\
+\Sigma\left|\frac{\partial \varphi}{\partial x_{i}}-\frac{\partial \varphi}{\partial y_{i}}\right|\left|\frac{\partial y_{i}}{\partial a}\right|+\left|\frac{\partial \varphi}{\partial t}-\frac{\partial \varphi}{\partial s}\right|\left|\frac{\partial s}{\partial a}\right|+K|p-q|+K\left|\frac{\partial p}{\partial a}-\frac{\partial q}{\partial a}\right|+K|\varphi(x, v, t, \varepsilon)-r(y, v, t, 0)|
\end{array}
$$

Let $v_{3}<v_{C}$. We observe that $v_{3}$ is unrelated to $v_{3}$ of Theorem 1. If $\psi\left(\varepsilon, \delta_{1}, \delta_{2}, v_{3}\right)$ represents a function which tends to zero as $\varepsilon, \delta_{1}$, and $\delta_{2} \rightarrow 0$ then for $v_{0} \leqq v \leqq v_{3}$

$$
\left|\frac{d z}{d v}\right| \leqq K|z|+K|p-q|+K\left|\frac{\partial p}{\partial a}-\frac{\partial q}{\partial a}\right|+\psi\left(\varepsilon, \delta_{1}, \delta_{2}, v_{3}\right)
$$


From Lemma 1

$$
|z(v)| \leqq e^{K\left(v-v_{0}\right)}\left[\left|z\left(v_{0}\right)\right|+K \int_{v_{0}}^{v}|p-q| d v+K \int_{v_{0}}^{v}\left|\frac{\partial p}{\partial a}-\frac{\partial q}{\partial a}\right| d v+\left(v-v_{0}\right) \psi\right]
$$

It follows easily from Theorem 1 that

$$
\int_{v_{0}}^{v_{3}}|p-q| d v \rightarrow 0
$$

as $\varepsilon, \delta_{1}$, and $\delta_{2} \rightarrow 0$. We can also make $\left|z\left(v_{0}\right)\right|$ as small as we wish. Thus the terms on the right side of (6.6) all go to zero as $\varepsilon, \delta_{1}, \delta_{2} \rightarrow 0$ with the possible exception of

$$
J=\int_{v_{0}}^{v_{3}}\left|\frac{\partial p}{\partial a}-\frac{\partial q}{\partial a}\right| d v
$$

Let $v_{0}<v_{1}<v_{B}$. Then since $q=0$ for $v_{B}<v<v_{C}$,

$$
J \leqq \int_{v_{0}}^{v_{1}}\left|\frac{\partial p}{\partial a}-\frac{\partial q}{\partial a}\right| d v+\int_{v_{1}}^{v_{B}}\left|\frac{\partial q}{\partial a}\right| d v+\int_{v_{1}}^{v_{3}}\left|\frac{\partial p}{\partial a}\right| d v .
$$

By taking $v_{1}$ close enough to $v_{B}$ and then taking $\varepsilon, \delta_{1}$, and $\delta_{2}$ small enough the first two terms on the right above can be made as small as we wish. Thus given any $\varepsilon_{3}>0$ we can make, by (6.6).

$$
|z(v)| \leqq \varepsilon_{3}+K \int_{v_{1}}^{v_{3}}\left|\frac{\partial p}{\partial a}\right| d v
$$

providing we have chosen $v_{0}$ near enough to $v_{B}$ so $|p|+|q| \leqq 1$ for $v_{0} \leqq v \leqq v_{3}$ and so long as $|z| \leqq 1$ and $v_{0} \leqq v \leqq v_{3}$. From $\varepsilon d p / d u=p^{2} g+p^{3} h$ we find

Thus

$$
\varepsilon \frac{d}{d u}\left(\frac{1}{p^{2}} \frac{\partial p}{\partial a}\right)-p^{2} h\left(\frac{1}{p^{2}} \frac{\partial p}{\partial a}\right)=\frac{\partial g}{\partial a}+p \frac{\partial h}{\partial a}
$$

$$
\frac{\partial p(v)}{\partial a}=p^{2}(v)\left(\frac{1}{p^{2}\left(v_{1}\right)} \frac{\partial p\left(v_{1}\right)}{\partial a}\right) e^{\frac{1}{\varepsilon} v_{v_{1}}^{v} p h d u}+\frac{p^{2}}{\varepsilon} \int_{v_{1}}^{v}\left(\frac{\partial g}{\partial a}+p^{\partial h}\right) e^{\frac{1}{\varepsilon} \int^{v} \sigma^{p 2 h d u}} d \sigma
$$

Let $v_{2}>v_{B}$ and let $\min |h| \geqq \mu>0$ near $B$. By (4.12), $p \leqq \varepsilon / \delta_{6} \cdot$ for $v_{2} \leqq v \leqq v_{3}$. By taking $\varepsilon, \delta_{1}$, and $\delta_{2}$ small enough we have then $p<\varepsilon^{3}$ for $v_{2} \leqq v \leqq v_{3}$. Since $h<0$ near $B$ we have for $v_{2} \leqq v \leqq v_{3}$,

$$
\int_{v_{1}}^{v} p^{2} e^{\frac{1}{\varepsilon}} v_{v_{1}}^{\sigma}{ }^{p 2 h d u} d \sigma \leqq \frac{\varepsilon}{\mu}+\int_{v_{2}}^{v} p^{2} e^{\frac{1}{\varepsilon} \int_{v_{1}}^{\sigma}{ }^{2 h d u}} d \sigma \leqq \frac{\varepsilon}{\mu}+K \varepsilon^{3 / 2}
$$

Also in much the same way 


$$
\begin{gathered}
\left|\int_{v_{1}}^{v} \frac{p^{2}}{\varepsilon} d u \int_{v_{1}}^{u}\left(\frac{\partial g}{\partial a}+p \frac{\partial h}{\partial a}\right) e^{\frac{1}{\varepsilon} \int_{\sigma}^{u} p^{2 h d u}} d \sigma\right|=\left|\int_{v_{1}}^{v}\left(\frac{\partial g}{\partial a}+p \frac{\partial h}{\partial a}\right) d \sigma \int_{\sigma}^{v} \frac{p^{2}}{\varepsilon} e^{\frac{1}{\varepsilon} \int_{\sigma}^{u} p^{2 h} d u} d u\right| \\
\leqq K \int_{v_{1}}^{v_{2}}(1+|z(\sigma)|) d \sigma\left(\frac{1}{\mu}+\varepsilon^{\frac{1}{2}}\right)+K \varepsilon^{\frac{1}{2}} \int_{v_{2}}^{v}(1+|z(\sigma)|) d \sigma
\end{gathered}
$$

From (6.8), (6.9) and (6.10)

$$
\int_{v_{1}}^{v_{3}}\left|\frac{\partial p(v)}{\partial a}\right| d v \leqq \varepsilon_{4}+\frac{K}{\mu} \int_{v_{1}}^{v_{2}}(1+|z(\sigma)|) d \sigma+K \varepsilon^{\frac{1}{2}} \int_{v_{1}}^{v_{3}}(1+|z(\sigma)|) d \sigma
$$

where for any choice of $v_{1}, \varepsilon_{4}$ can be made as small as we wish by taking $\varepsilon, \delta_{1}$, and $\delta_{2}$ small enough. Thus (6.7) yeilds, for arbitrary $\varepsilon_{5}$,

$$
|z(v)| \leqq \varepsilon_{5}+\frac{K\left(v_{2}-v_{1}\right)}{\mu}+\frac{K}{\mu} \int_{v_{1}}^{v_{2}}|z(\sigma)| d \sigma+K \varepsilon^{\frac{1}{2}} \int_{v_{1}}^{v_{3}}|z(\sigma)| d \sigma
$$

Let $v_{2}-v_{1}$ be small enough so that $K\left(v_{2}-v_{1}\right) / \mu<\varepsilon_{6}$ where $\varepsilon_{6}$ is a preassigned positive quantity. Taking $v_{1}$ and $v_{2}$ closer to $v_{B}$ affects the previous argument only in so far as it may be necessary to decrease $\varepsilon, \delta_{1}$ and $\delta_{2}$. Let $\max |z(v)|=M, v_{1} \leqq v \leqq v_{3}$. (If $M \geqq 1$ decrease $v_{3}$ so that we get $M<1$ ). Then from

or

$$
M \leqq \varepsilon_{5}+\varepsilon_{6}+M \varepsilon_{6}+K \varepsilon^{\frac{1}{2}} M
$$

$$
M\left(1-\varepsilon_{6}-K \varepsilon^{\frac{1}{2}}\right) \leqq \varepsilon_{5}+\varepsilon_{6} .
$$

Thus $2\left(\varepsilon_{5}+\varepsilon_{6}\right) \geqq M \geqq|z(v)|$. (In particular then it is unecessary to decrease $v_{3}$ to achieve $M<1$ ). We see then that $(6.11)$ becomes, for any preassigned $\varepsilon_{7}>0$,

and thus from $(6.7)$

$$
\int_{v_{1}}^{v_{3}}\left|\frac{\partial p(v)}{\partial a}\right| d v \leqq \varepsilon_{7}
$$

for any preassigned $\varepsilon_{8}$. Thus $\frac{\partial x}{\partial a}-\frac{\partial y}{\partial a}$ and $\frac{\partial t}{\partial a}-\frac{\partial s}{\partial a}$ tend to zero uniformly over $v_{0} \leqq v$ $\leqq v_{3}$ as $\varepsilon, \delta_{1}$, and $\delta_{2}$ tend to zero. That is we have established Theorem 3 up to any point short of $C$.

The case $\partial x / \partial b, \partial t / \partial b$ and $\partial p / \partial b$ is handled with the usual modification. That is we now set $z=\left(\frac{\partial x}{\partial b}, \frac{\partial t}{\partial b}\right)$ and use the fact that at $v_{0}$ the values of $|z|$ and $\partial p / \partial b$ tend to zero as $\varepsilon \rightarrow 0$.

7. Acta mathematica, 82. Imprimé le 14 janvier 1950. 
We now turn to the proof of Lemma 4 .

Proof of Lemma 4. Actually the result of this lemma is a consequence of Part 1 of Theorem 3 which we have already proved. There we have shown that

$$
\frac{d}{d t} \frac{\partial u}{\partial a}-\frac{d}{d t} \frac{\partial v}{\partial a} \rightarrow 0, \quad \alpha+\delta \leqq t \leqq \gamma,
$$

uniformly as $\varepsilon, \delta_{1}$ and $\delta_{2} \rightarrow 0$. By a well known artifice $\partial / \partial a$ can be changed to $d / d t$. Indeed replace $t$ by $\sigma+\alpha$ in (1.2). Then we have

$$
\frac{d t}{d \sigma}=1, \quad \frac{d x}{d \sigma}=f(x, u, t, \varepsilon) \frac{d u}{d \sigma}+\varphi,
$$

$$
\frac{d^{2} u}{d \sigma^{2}}+g \frac{d u}{d \sigma}+h=0
$$

Here we have one more dependent variable than in (1.2) namely $t$. The independent variable $\sigma$ is assigned the intitial value $\sigma=0$. Clearly then $t(0)=\alpha$ and $\alpha$ is an $a$ for (6.16). Also $u=u\left(\sigma+\alpha, \varepsilon, x(0), u(0), u^{\prime}(0)\right)$ where $x(0)$ etc. are the values of $x$ etc. at $\sigma=0$. Clearly $\frac{\partial u}{\partial \alpha}=\frac{d u}{d \sigma}=\frac{d u}{d t}$ and similarly for $v$. Using Part 1 of Theorem 1 for the system (6.16) we have then the proof of Lemma 4 as a consequence of (6.15).

The proof of Lemma 4 for $\tau_{1}+\delta \leqq t \leqq \beta$ follows in the same way once (6.15) is demonstrated over this range of $t$.

\section{Theorem 3, Part 3.}

Before discussing the behavior of $\partial x / \partial a, \partial t / \partial a$ and $\partial p / \partial a$ near $C$ it is convenient to obtain the following result

$$
\frac{\varepsilon}{p^{2}} \frac{\partial p}{\partial a}-\int_{v_{B}}^{v} \frac{\partial g}{\partial a} d v \rightarrow 0
$$

uniformly for $v_{B} \leqq v \leqq v_{C}-\delta$ for $\delta>0$, as $\varepsilon, \delta_{1}$, and $\delta_{2} \rightarrow 0$. In (7.0)

$$
\frac{\partial g}{\partial a}=\Sigma \frac{\partial g}{\partial x_{i}} \frac{\partial x_{i}}{\partial a}+\frac{\partial g}{\partial t} \frac{\partial t}{\partial a}
$$

The proof is a consequence of integrating

$$
\frac{d}{d u}\left(\frac{\varepsilon}{p^{2}} \frac{\partial p}{\partial a}\right)=h \frac{\partial p}{\partial a}+\frac{\partial g}{\partial a}+p \frac{\partial h}{\partial a}
$$

over $v_{1} \leqq u \leqq v \leqq v_{C}-\delta$ and using (6.13) and the fact that $p \rightarrow 0$ uniformly in the interval $v_{B} \leqq u \leqq v_{C}-\delta$ as $\varepsilon, \delta_{1}, \delta_{2} \rightarrow 0$. 
We take $v_{3}<v_{C}$ and near $v_{C}$ and choose the point $P_{4}\left(x_{4}, u_{4}, t_{4}\right)$ so that $P_{4}$ is the first point where we have $p=p_{4}=\varepsilon^{\frac{1}{4}}$ and $t_{4}>t_{3} . P_{4}$ here is unrelated to the point $P_{4}$ used in the proof of Theorem 1. Clearly from (4.12) of Theorem $1, P_{4} \rightarrow C$ as $\varepsilon, \delta_{1}, \delta_{2} \rightarrow 0$.

From (6.4), letting $z=\left(\frac{\partial x}{\partial a}, \frac{\partial t}{\partial a}\right)$ we have

$$
\left|\frac{d z}{d u}\right| \leqq K|z|+K|z p|+K\left|\frac{\partial p}{\partial a}\right| \leqq K|z|+K\left|\frac{\partial p}{\partial a}\right|, v_{3} \leqq u \leqq v_{4},
$$

and therefore by Lemma 1

$$
|z(u)| \leqq K\left|z_{3}\right|+K \int_{v_{3}}^{u}\left|\frac{\partial p}{\partial a}\right| d u, v_{3} \leqq u \leqq v_{4} .
$$

We have since $g>0$ near $C, g+p h>\frac{1}{2} g, v_{3} \leqq u \leqq v_{4}$, if we have chosen $\varepsilon$ small enough. Thus from $\varepsilon d p / d u=g p^{2}+h p^{3}, p$ is an increasing function of $u$. Also $d p>\frac{1}{2} g p^{2} d u / \varepsilon$ so that

$$
\frac{1}{\varepsilon} \int_{v_{3}}^{v_{4}} p^{2} d u<K\left(p_{4}-p_{3}\right)<K p_{4}
$$

From (6.8)

$$
\frac{\partial p(u)}{\partial a}=\frac{p^{2}(u)}{\varepsilon}\left(\frac{\varepsilon}{p_{3}^{2}} \frac{\partial p\left(v_{3}\right)}{\partial a}\right) e^{\frac{1}{\varepsilon} \int_{v_{3}}^{u} p 2 h d u}+\frac{p^{2}(u)}{\varepsilon} \int_{v_{3}}^{u}\left(\frac{\partial g}{\partial a}+p \frac{\partial h}{\partial a}\right) e^{\frac{1}{\varepsilon}} \int_{\sigma}^{u} p 2 h d v d \sigma
$$

Using the fact that $\frac{\varepsilon}{p_{3}^{2}} \frac{\partial p\left(v_{3}\right)}{\partial a}$ is bounded by (7.0) and also using (7.2) we find easily now that for $v_{3} \leqq v \leqq v_{4}$

$$
\int_{v_{3}}^{v}\left|\frac{\partial p}{\partial a}\right| d u \leqq K p_{4}+K p_{4} \int_{v_{3}}^{v}|z| d u, \quad \varepsilon+\delta_{1}+\delta_{2}<\Psi\left(v_{3}\right)
$$

where $\Psi\left(v_{3}\right)$ is a continuous function which tends to zero as $v_{3} \rightarrow v_{C}$. With the formula below (7.1) this yields

$$
|z(u)| \leqq M \leqq K\left|z_{3}\right|+K p_{4}+K p_{4} M\left(v_{4}-v_{3}\right), \quad \varepsilon+\delta_{1}+\delta_{2}<\Psi\left(v_{3}\right)
$$

where $M=\max |z(u)|, v_{3} \leqq u \leqq v_{4}$. Thus if $\varepsilon$ is small enough $M \leqq K\left|z_{3}\right|+K p_{4}<K$ since $z_{3}$ is near $\left(\frac{\partial y\left(v_{3}\right)}{\partial a}, \frac{\partial \tau_{1}}{\partial a}\right)$.

Since $|z(u)|<K$ for $v_{B} \leqq v \leqq v_{3}$ we have

$$
|z(u)| \leqq K, \quad v_{B} \leqq u \leqq v_{4} .
$$


By (7.4) and (7.5) we find from (7.1)

$$
\left|z_{4}-z_{3}\right| \leqq K\left(v_{4}-v_{3}\right)+K p_{4}, \quad \varepsilon+\delta_{1}+\delta_{2}<\Psi\left(v_{3}\right) .
$$

At $P_{4}$ we change again from $u$ to $t$ as the independent variable. We have, if $t=t_{4}$ at $P_{4}$, from $t(u, a)=t_{4}$

$$
\frac{\partial u\left(t_{4}\right)}{\partial a}=-\frac{\partial t\left(u_{4}\right)}{\partial a} \frac{d u\left(t_{4}\right)}{d t}
$$

where $\partial u\left(t_{4}\right) / \partial a$ is $\partial u(t) / \partial a$ at $t=t_{4}+0$, etc. analogous to (5.1) and (5.2). We also have

$$
\frac{\partial x\left(t_{4}\right)}{\partial a}=\frac{\partial x\left(u_{4}\right)}{\partial a}-\frac{d x\left(t_{4}\right)}{d t} \frac{\partial t\left(u_{4}\right)}{\partial a}
$$

$$
\frac{\partial}{\partial a} \frac{d u\left(t_{4}\right)}{d t}=-\frac{1}{p_{4}^{2}} \frac{\partial p\left(u_{4}\right)}{\partial a}+\frac{1}{p_{4}^{3}} \frac{d p\left(u_{4}\right)}{d u} \frac{\partial t\left(u_{4}\right)}{\partial a} .
$$

We define $t_{5}=t_{4}+\varepsilon \log ^{2} \frac{1}{\varepsilon}$ and denote the point $\left(x_{5}, u_{5}, t_{5}\right)$ by $P_{5}$. We have from (1.2)

$$
\frac{d u(t)}{d t}=\frac{d u\left(t_{4}\right)}{d t} e^{-\frac{1}{\varepsilon} \int_{t_{4}}^{t} g d t}-\frac{1}{\varepsilon} \int_{t_{4}}^{t} h e^{-\frac{1}{\varepsilon} \int_{\sigma^{2}}^{t} g d \sigma} d \sigma
$$

It follows easily that by integrating

by parts we have for small $\varepsilon$

$$
\int_{t_{4}}^{t}\left(\frac{h}{g}\right) g e^{-\frac{1}{\varepsilon} \int_{\sigma}^{t g d \sigma}} d \sigma
$$

$$
\left|\frac{d u\left(t_{5}\right)}{d t}+\frac{h_{5}}{g_{5}}\right| \leqq \frac{\varepsilon}{p_{4}}+K\left(t_{5}-t_{4}\right)<\varepsilon^{\frac{1}{2}}
$$

where $h_{5}$ denotes $h(x, u, t, \varepsilon)$ at $P_{5}$ etc.

By (6.0) and Lemma 1 if $\bar{z}=\left(\frac{\partial x(t)}{\partial a}, \frac{\partial u(t)}{\partial a}\right)$ then for $t_{4} \leqq t \leqq t_{5}$

$$
|\bar{z}| \leqq\left|\bar{z}_{4}\right| e^{K \int_{t_{4}}^{t}\left|\frac{d u}{d t}\right| d t+K\left(t-t_{4}\right)}+K \int_{t_{4}}^{t}\left|\frac{d}{d \sigma} \frac{\partial u}{\partial a}\right| e^{K \int_{\sigma}^{t}\left|\frac{d u}{d t}\right| d t+K(t-\sigma)} d \sigma
$$

From (7.9) we have easily if $g \geqq K_{1}>0$ near $C$ where $K_{1}$ is clearly a $K$,

$$
\left|\frac{d u}{d t}\right| \leqq \frac{1}{p_{4}} e^{-K_{1}\left(t-t_{9}\right) \varepsilon}+K
$$


Thus for $t_{4} \leqq t \leqq t_{5}$

Also from

$$
\int_{t_{4}}^{t}\left|\frac{d u}{d t}\right| d t \leqq \frac{K \varepsilon}{p_{4}}+K\left(t_{5}-t_{4}\right) \leqq \frac{K \varepsilon}{p_{4}}
$$

$$
\begin{gathered}
\frac{d}{d t} \frac{\partial u}{\partial a}=\frac{d}{d t} \frac{\partial u\left(t_{4}\right)}{\partial a} e^{-\frac{1}{\varepsilon} \int_{t_{4}}^{t} g d t}-\frac{1}{\varepsilon} \int_{t_{4}}^{t}\left(\frac{\partial g}{\partial a} \frac{d u}{d \sigma}+\frac{\partial h}{\partial a}\right) e^{-\frac{1}{\varepsilon} \int_{\sigma}^{t} g d \sigma} d \sigma \\
\int_{t_{4}}^{t}\left|\frac{d}{d t} \frac{\partial u}{\partial a}\right| d t \leqq K \varepsilon\left|\frac{d}{d t} \frac{\partial u\left(t_{4}\right)}{\partial a}\right|+K \int_{t_{4}}^{t}|\bar{z}|\left(\left|\frac{d u}{d t}\right|+1\right) d t
\end{gathered}
$$

From (7.2), (7.3) and (7.5) we have easily that since $\frac{\varepsilon}{p_{3}^{2}} \frac{\partial p\left(u_{3}\right)}{\partial a}$ is bounded by (7.0) as $\varepsilon, \delta_{1}, \delta_{2} \rightarrow 0$

$$
\left|\frac{\varepsilon}{p_{4}^{2}} \frac{\partial p\left(u_{4}\right)}{\partial a}\right| \leqq K
$$

From (7.8) then and (7.5) and (4.3)

$$
\varepsilon\left|\frac{\partial}{\partial a} \frac{d u\left(t_{4}\right)}{d t}\right| \leqq K+\frac{K}{p_{4}}<\frac{K}{p_{4}}
$$

Using the above, (7.13) and (7.15) in (7.11) we have if $\max |\bar{z}|=M$ for $t_{4} \leqq t \leqq t_{5}$ and if $\varepsilon$ is small enough,

$$
M \leqq K\left|\vec{z}_{4}\right|+\frac{K}{p_{4}}+K M\left(\frac{\varepsilon}{p_{4}}+t_{5}-t_{4}\right) .
$$

Since $K\left(\frac{\varepsilon}{p_{4}}+t_{5}-t_{4}\right)<\frac{1}{2}$ if $\varepsilon$ is small we have

$$
|\bar{z}(t)| \leqq K\left|\bar{z}_{4}\right|+\frac{K}{p_{4}} .
$$

Using (7.5), (7.7) and (7.8), we have $\left|\bar{z}_{4}\right| \leqq K / p_{4}$ and thus

$$
\left|\frac{\partial u(t)}{\partial a}\right|+\left|\frac{\partial x(t)}{\partial a}\right| \leqq \frac{K}{p_{4}}, \quad t_{4} \leqq t \leqq t_{5} .
$$

By integrating (7.14),

where

$$
\frac{\partial u(t)}{\partial a}=\frac{\partial u\left(t_{4}\right)}{\partial a}+\frac{d}{d t} \frac{\partial u\left(t_{4}\right)}{\partial a} \int_{t_{4}}^{t} e^{-\frac{1}{\epsilon} \sigma_{t_{4}}^{\sigma} g d \sigma} d \sigma-J_{1}
$$

$$
J_{1}=\frac{1}{\varepsilon} \int_{i_{4}}^{t}\left(\frac{d u}{d t} \frac{\partial g}{\partial a}+\frac{\partial h}{\partial a}\right) d \sigma \int_{\sigma}^{t} e^{-\frac{1}{\varepsilon} \int_{\sigma}^{g} g d \sigma} d s .
$$


Using (7.7) and (7.8)

(7.17) $\frac{\partial u(t)}{\partial a}=-\frac{1}{p_{4}} \frac{\partial t\left(u_{4}\right)}{\partial a}+\left[-\frac{1}{p_{4}^{2}} \frac{\partial p\left(u_{4}\right)}{\partial a}+\frac{1}{p_{4}^{3}} \frac{d p\left(u_{4}\right)}{d u} \frac{\partial t\left(u_{4}\right)}{\partial a}\right] \int_{t_{4}}^{t} e^{-\frac{1}{\varepsilon} \int_{t_{4}}^{\sigma} g d t} d \sigma-J_{1}$.

Clearly using (7.16) and (7.13) for $t_{4} \leqq t \leqq t_{5}$ we find

$$
\left|J_{1}\right| \leqq \frac{K}{p_{4}} \int_{t_{4}}^{t_{5}}\left(\left|\frac{d u}{d t}\right|+1\right) d t \leqq \frac{K \varepsilon}{p_{4}^{2}}<\varepsilon^{\frac{1}{4}}
$$

for small $\varepsilon$. Integrating by parts we have

where

$$
\int_{t_{4}}^{t_{5}} \frac{1}{g}\left(g e^{-\frac{1}{\varepsilon} \int_{t_{4}}^{\sigma} g d t}\right) d \sigma=\frac{\varepsilon+J_{2}}{g_{4}}
$$

$$
\left|J_{2}\right|<\varepsilon^{\frac{9}{2}} .
$$

Thus from (7.17)

$$
\frac{\partial u\left(t_{5}\right)}{\partial a}=-\frac{1}{p_{4}} \frac{\partial t\left(u_{4}\right)}{\partial a}+\frac{\varepsilon}{g_{4} p_{4}^{2}}\left[-\frac{\partial p\left(u_{4}\right)}{\partial a}+\frac{1}{p_{4}} \frac{d p\left(u_{4}\right)}{d u} \frac{\partial t\left(u_{4}\right)}{\partial a}\right]\left(1+\frac{1}{\varepsilon} J_{2}\right)-J_{1} .
$$

Since $\varepsilon d p / d u=p^{2} g+p^{3} h$,

$$
\frac{\partial u\left(t_{5}\right)}{\partial a}=\left[\frac{h_{4}}{g_{4}} \frac{\partial t\left(u_{4}\right)}{\partial a}-\frac{\varepsilon}{g_{4} p_{3}^{2}} \frac{\partial p\left(u_{3}\right)}{\partial a}\right]\left(1+\frac{1}{\varepsilon} J_{2}\right)+\frac{1}{\varepsilon} J_{2} \frac{1}{p_{4}} \frac{\partial t\left(u_{4}\right)}{\partial a}+J_{3}-J_{1}
$$

where

$$
J_{3}=\frac{1}{g_{4}}\left(\frac{\varepsilon}{p_{3}^{2}} \frac{\partial p\left(u_{3}\right)}{\partial a}-\frac{\varepsilon}{p_{4}^{2}} \frac{\partial p\left(u_{4}\right)}{\partial a}\right)\left(1+\frac{1}{\varepsilon} J_{2}\right) .
$$

From (7.3) and (7.2) we have also using (7.0)

$$
\left|\frac{\varepsilon}{p_{4}^{2}} \frac{\partial p\left(u_{4}\right)}{\partial a}-\frac{\varepsilon}{p_{3}^{2}} \frac{\partial p\left(u_{3}\right)}{\partial a}\right| \leqq K p_{4} \frac{\varepsilon}{p_{3}^{2}}\left|\frac{\partial p\left(u_{3}\right)}{\partial a}\right|+K\left(v_{4}-v_{3}\right) .
$$

Since $v_{3}$ and $v_{4}$ can be as near $v_{C}$ as we wish we see that by taking $\varepsilon, \delta_{1}$, and $\delta_{2}$ small enough we can make

$$
\left|J_{3}\right|<\varepsilon_{9}
$$

where $\varepsilon_{9}>0$ is any prescribed quantity. By (7.6)

Thus (7.20) yields

$$
\left|\frac{\partial t\left(u_{4}\right)}{\partial a}-\frac{\partial t\left(u_{3}\right)}{\partial a}\right| \leqq K p_{4}+K\left(v_{4}-v_{3}\right) .
$$

$$
\frac{\partial u\left(t_{5}\right)}{\partial a}=\frac{h_{4}}{g_{4}} \frac{\partial t\left(u_{3}\right)}{\partial a}-\frac{\varepsilon}{g_{4} p_{3}^{2}} \frac{\partial p\left(u_{3}\right)}{\partial a}+\varepsilon_{10}
$$


Perturbations of Discontinuous Solutions of Non-Linear Syst. of Diff. Equations. 103 where $\varepsilon_{10}>0$ can be chosen arbitrarily small. From (7.0) and continuity considerations the above formula yield

$$
\left|\frac{\partial u\left(t_{5}\right)}{\partial a}-\frac{h_{C} \partial \tau_{1}}{g_{C}} \partial \frac{1}{\partial a} \int_{v_{v_{B}}}^{v_{C}} \frac{\partial g}{\partial a} d v\right|<\varepsilon_{11}
$$

where $\frac{\partial g}{\partial a}=\sum \frac{\partial g}{\partial y_{i}} \frac{\partial y_{i}}{\partial a}+\frac{\partial g}{\partial s} \frac{\partial \tau_{1}}{\partial a}$ and where $\varepsilon_{11}>0$ can be chosen as small as we wish by taking $\varepsilon, \delta_{1}$, and $\delta_{2}$ small enough. Comparing with (5.9) we have

$$
\left|\frac{\partial u\left(t_{5}\right)}{\partial a}-\frac{\partial v\left(t_{C}\right)}{\partial a}\right|<\varepsilon_{11}
$$

From (7.14), if $g_{C}>2 K_{1}>0$, we have using (7.8), (7.12), (7.16) and (7.21)

$$
\left|\frac{d}{d t} \frac{\partial u\left(t_{5}\right)}{\partial a}\right| \leqq \frac{K}{\varepsilon p_{4}} e^{-\left(t_{5}-t_{4}\right) K_{1} / \varepsilon}+\frac{K}{p_{4}^{2}}<\frac{\varepsilon^{1}}{\varepsilon}
$$

for small $\varepsilon, \delta_{1}$, and $\delta_{2}$.

Finally from $(6.0)$

$$
\frac{\partial}{d t} \frac{\partial x}{\partial a}=\frac{d}{d t}\left(f \frac{\partial u}{\partial a}\right)-\frac{\partial u}{\partial a} \frac{d f}{d t}+\frac{\partial \varphi}{\partial a}+\frac{\partial f}{\partial a} \frac{d u}{d t}
$$

Integrating we have

$$
\frac{\partial x\left(t_{5}\right)}{\partial a}=\frac{\partial x\left(t_{4}\right)}{\partial a}+f_{5} \frac{\partial u\left(t_{5}\right)}{\partial a}-f_{4} \frac{\partial u\left(t_{4}\right)}{\partial a}+J
$$

where for small $\varepsilon, \delta_{1}$, and $\delta_{2}$, by (7.16) and (7.13)

Or by (7.7) and (7.8)

$$
|J|<\frac{K \varepsilon}{p_{4}^{2}}<\varepsilon^{1} .
$$

$$
\begin{gathered}
\frac{\partial x\left(t_{5}\right)}{\partial a}=\frac{\partial x\left(u_{4}\right)}{\partial a}-\frac{d x\left(t_{4}\right)}{d t} \frac{\partial t\left(u_{4}\right)}{\partial a}+f_{5} \frac{\partial u\left(t_{5}\right)}{\partial a}+f_{4} \frac{d u\left(t_{4}\right)}{d t} \frac{\partial t\left(u_{4}\right)}{\partial a}+J \\
=\frac{\partial x\left(u_{4}\right)}{\partial a}+f_{5} \frac{\partial u\left(t_{5}\right)}{\partial a}-\varphi_{4} \frac{\partial t\left(u_{4}\right)}{\partial a}+J .
\end{gathered}
$$

Or by $(7.6),(7,23)$ and continuity considerations

$$
\left|\frac{\partial x\left(t_{5}\right)}{\partial a}-\frac{\partial x\left(u_{3}\right)}{\partial a}-f_{C} \frac{\partial u\left(t_{5}\right)}{\partial a}+\varphi_{C} \frac{\partial t\left(u_{3}\right)}{\partial a}\right|<\varepsilon_{12}
$$

where $\varepsilon_{12}>0$ can be chosen less than any prescribed quantity by taking $\varepsilon, \delta_{1}$, and $\delta_{2}$ small enough. Or by Theorem 3, part 2 and by (7.23) 


$$
\left|\frac{\partial x\left(t_{5}\right)}{\partial a}-\frac{\partial y\left(v_{C}\right)}{\partial a}-f_{C} \frac{\partial v\left(t_{C}\right)}{\partial a}+\varphi_{C} \frac{\partial \dot{\tau}_{1}}{\partial a}\right|<\varepsilon_{\mathbf{1 3}}
$$

where $\varepsilon_{13}>0$ etc. Comparing with (5.10) we have now

$$
\left|\frac{\partial x\left(t_{5}\right)}{\partial a}-\frac{\partial y\left(t_{C}\right)}{\partial a}\right|+\left|\frac{\partial u\left(t_{5}\right)}{\partial a}-\frac{\partial v\left(t_{C}\right)}{\partial a}\right|
$$

as small as we wish. With (7.24), (7.26) and (7.10) we can proceed now as in Part I from $t_{5}$ to $\beta$ since in the range $t_{C}$ to $t_{5}$ or $t_{5}$ to $t_{C}$ the change in the degenerate system is small because $\left|t_{5}-t_{C}\right|$ is small.

The case where $b$ is used instead of $a$ proceeds with the usual modifications. This completes the proof of Theorem 3 .

8. Let us consider the case where the degenerate system has a solution, as defined in $\S 2$, which is periodic of period $T$. Let $t=\alpha$ be a point where $g \neq 0$. Suppose in the first place we take the case where the functions $f(x, u, t, \varepsilon), \varphi, g$ and $h$ are periodic in $t$ of period $T$. Let the initial values for (1.3) be $y_{1}(\alpha), \ldots, y_{n}(\alpha), v(\alpha)$. Let us denote the initial values of $x_{1}(\alpha), \ldots, x_{n}(\alpha), u(\alpha), \frac{d u(\alpha)}{d t}$ by $a_{1}, \ldots, a_{n+1}, b$. Then for the system (1.2) to have a periodic solution for small $\varepsilon$ it suffices that, if $\beta=\alpha+T$, the determinant at $t=\beta$,

$$
D\left(a_{1}, \ldots, a_{n+1}, b, \varepsilon\right)=\left|\begin{array}{ccccc}
\frac{\partial x_{1}}{\partial a_{1}}-1 & \frac{\partial x_{1}}{\partial a_{2}} & \ldots & \frac{\partial x_{1}}{\partial a_{n+1}} & \frac{\partial x_{1}}{\partial b} \\
\frac{\partial x_{2}}{\partial a_{1}} \frac{\partial x_{2}}{\partial a_{2}}-1 & \ldots & . & \frac{\partial x_{2}}{\partial b} \\
\cdot & . & \ldots & \cdot & \cdot \\
\frac{\partial u}{\partial a_{1}} & . & \ldots & \frac{\partial u}{\partial a_{n+1}}-1 & \frac{\partial u}{\partial b} \\
\frac{\partial}{\partial a_{1}} \frac{d u}{d t} & . & \ldots & . & \frac{\partial}{\partial b} \frac{d u}{d t}-1
\end{array}\right|
$$

should be continuous as

$\varepsilon \rightarrow+0 ; a_{1}, \ldots, a_{n+1} \rightarrow y_{1}(\alpha), \ldots, v(\alpha) ;$ and $b \rightarrow-\frac{h_{A}}{g_{A}}$, and $D$ should not vanish.

This is a consequence of the fact that the existence of a periodic solution of (1.2) is equivalent to the existence of a solution $\left(a_{1}, \ldots, a_{n+1}, b\right)$ of 


$$
\begin{gathered}
x_{i}\left(\beta, a_{1}, \ldots, a_{n+1}, b, \varepsilon\right)=a_{i}, i=1,2, \ldots, n, \\
u\left(\beta, a_{1}, \ldots, a_{n+1}, b, \varepsilon\right)=a_{n+1}, \frac{d u\left(\beta, a_{1}, \ldots, a_{n+1}, b, \varepsilon\right)}{d t}=b,
\end{gathered}
$$

where $\left(a_{1}, \ldots, a_{n+1}, b\right)$ are found as functions of $\varepsilon>0$. By Theorem 3 , the last column of $D$ tends to zero except for the last term in the column which tends to -1 . Thus $D$ tends to

$$
-\left|\begin{array}{cccc}
\frac{\partial y_{1}(\beta)}{\partial a_{1}}-1 & \frac{\partial y_{1}(\beta)}{\partial a_{2}} & \cdots & \frac{\partial y_{1}(\beta)}{\partial a_{n+1}} \\
\cdot & \cdot & \cdots & \cdot \\
\frac{\partial v(\beta)}{\partial a_{n+1}} & \cdot & \cdots & \frac{\partial v(\beta)}{\partial a_{n+1}}-1
\end{array}\right|
$$

as $\varepsilon, \delta_{1}$, and $\delta_{2} \rightarrow 0$. This is the Jacobian associated with a periodic solution of (1.3) and if it is different from zero we see that (1.2) also has a unique nearby periodic solution for small $\varepsilon>0$. In particular if the periodic solution of (1.3) is stable in the sense that the associated characteristic roots are all less than one in magnitude then the determinant (8.0) does not vanish.

In case the right members of (1.2) do not involve $t$ the period $T$ is no longer a constant for periodic solutions (if any) of the perturbed system. Making the usual modification for this situation the same result relating the existence of periodic solutions for (1.2) to (1.3) holds again.

The case where the last equation of (1.2) is a vector equation can be treated quite readily on the basis of results obtained here by making a change of coordinates. We shall return to this case later. 


\section{REFERENCES}

1. D. A. Flanders and J. J. Stoker, The limit case of relaxation oscillations, Studies in nonlinear vibration theory, New York University, 1946.

2. K. O. Friedrichs and W. R. WASOW, Singular perturbations of non-linear oscillations, Duke Math Journal, 13, 367--381 (1946).

3. N. Irevinson, Perturbations of discontinuous solutions of non-linear systems of differential equations, Proc. Nat. Acad. of Sciences, 33, 214--218 (1947).

4. M. Nagumo, Über das Verhalten des Integrals von $\lambda y^{\prime \prime}+f\left(x, y, y^{\prime}, \lambda\right)=0$ für $\lambda \rightarrow 0$. Proc. Phys. Math. Soc., Japan 21, 529-534 (1939).

5. A. Trhonov, On the dependence of the solutions of differential equations on a small parameter. Mat. Sbornik N. S. 22, 193-204 (1948).

6. I. M. VoLk, A generalization of the method of small parameter in the theory of non-linear oscillations of non-autonomous systoms, C. R. (Doklady) Acad. Sci. U.S.S.R., 51, 437-440 (1946). 